\title{
Evolution of Tagged Particles in Non-Reversible Particle Systems
}

\section{Fraydoun Rezakhanlou*}

Department of Mathematics, University of California, Berkeley, California 94720, USA

Received: 24 May 1993

Abstract: We derive an ODE for the macroscopic evolution of a tagged particle in models such as asymmetric simple exclusions and zero range processes. The right-hand side of the ODE is discontinuous and its solutions are understood in the Filippov sense. We establish the uniqueness of the ODE, and explore its relationship with the hydrodynamic equation of the particle density.

\section{Introduction}

Imagine that every particle in a fluid moves with the velocity $b(x, t)$. In other words, if $x(t)$ is the path traversed by a particle in the fluid, we have

$$
\frac{d x}{d t}=b(x(t), t)
$$

If no particle is destroyed or created, one derives the transport equation

$$
\frac{\partial \rho}{\partial t}+\operatorname{div}(b \rho)=0
$$

for the macroscopic particle density $\rho(x, t)$.

In general one needs to employ the other conservation laws, such as the conservation of momentum and energy, to determine the velocity field $b$. If, however, the total number of particles is the only microscopically conserved quantity, it must be possible to express the velocity in terms of the particle density. In this case (1.2) would look like

$$
\frac{\partial \rho}{\partial t}+\operatorname{div}(F(\rho))=0
$$

‡ Research partially supported by National Science Foundation grant DMS-9208490. 
for a vector valued function $F$ and, as a result,

$$
b(x, t)=\frac{F(\rho(x, t))}{\rho(x, t)} .
$$

There is a class of particle systems for which an equation like (1.3) has been derived and we would like to derive an equation of the form (1.1) for the evolution of each particle in the system.

The Simple Exclusion Process (SEP) is one of the particle systems for which we will derive Eq. (1.1) with $F(\rho)=\gamma \rho(1-\rho)$ and $b(x, t)=\gamma(1-\rho(x, t))$, where $\gamma$ is a constant vector and $\rho(x, t)$ is a solution to (1.3).

Roughly speaking, SEP is a continuous time particle system in which particles move as random walks on a $d$-dimensional lattice but are excluded from occupying the same site. The precise definition of SEP will be given in the next section.

If the system consists of a single particle, it moves as a simple random walk with mean $\gamma$. The Law of Large Numbers for a simple random walk implies that $L^{-1}$ times the position of the particle at time $t L$ converges to $x(0)+t \gamma$, as $L$ goes to infinity. Here $x(0)$ is the initial macroscopic location of the particle. In particular this suggests scaling time by a factor of order $L$ and scaling space of order $L^{-1}$. If the system consists of a large number of particles that has, after the same space and time scaling, a macroscopic particle density $\rho(x, t)$ then, because of the exclusion rule, the speed of a tagged particle in the system will slow down to $\gamma(1-\rho(x, t))$.

Zero Range Process (ZRP) is another particle system for which Eq. (1.3) has been derived with $F(\rho)=\gamma h(\rho)$, where $\gamma$ is a constant vector and $h$ is a scalar valued function. In this model particles move on a lattice where the rate of each jump depends on the occupation number of the site the particle is jumping from, and the location of the site it is jumping to. See [10] and references therein.

It is well known that Eq. (1.3) does not in general possess globally defined smooth solutions. The nonlinearity of $F$ leads to the development of discontinuities in the solution.

It is necessary to interpret (1.3) in the distributional sense. Since there are infinitely many distributional solutions to (1.3) that share the same initial data, some additional conditions to the solutions are needed to ensure uniqueness. Kružkov [7] proposed the following entropy criterion: for every pair $(\phi, q)$ with $\phi$ convex and $q$ satisfying $\phi^{\prime} F^{\prime}=q^{\prime}$, we assume

$$
\frac{\partial}{\partial t} \phi(\rho)+\operatorname{div}(q(\rho)) \leqq 0
$$

in distributional sense. We will say a solution $\rho$ is an entropy solution if (1.5) holds for all pairs $(\phi, q)$.

The discontinuity of $b(x, t)$, inherited from $\rho(x, t)$ poses the problem of nonuniqueness for the initial value problem (1.1). A solution to (1.1) is understood in the Filippov sense [3]: an absolutely continuous function $x$ is a solution if for almost all $t, \frac{d x}{d t}(t)$ is between the essential infimum and the essential supremum of $b$ at the point $(x(t), t)$. See Sect. 2 for more details.

In Sect. 5, the precise form of $b(x, t)$ as in (1.4) will be used to establish the uniqueness of (1.1). 
We will assume that particles are initially located independently on the lattice. If the macroscopic particle density is $\rho_{0}(x)$, then the probability density of the position of each particle must be $c \rho_{0}(x)$, where $c$ is the normalizing constant.

The main result of this article asserts that the macroscopic evolution of a tagged particle satisfies (1.1) with the random initial condition

$$
\text { Prob. }(x(0) \in A)=c \int_{A} \rho_{0}(x) d x .
$$

If the particles move on a one-dimensional lattice and they are allowed to jump to adjacent sites only, we strengthen our results by deriving (1.1) with initial condition $x(0)=a$, for a tagged particle that is conditioned to start from $a$. For this we also assume that the initial density $\rho_{0}(x)$ is positive in a neighborhood of $a$.

If in any of the above systems the particles are initially distributed according to an equilibrium measure, then $\rho(x, t)$ is a constant and in the case of SEP, the problem was treated by Kipnis [5] and Saada $[13,14]$. They prove the law of large number for the position of the tagged particle even if the particle is conditioned to start from a fixed point.

We expect that the microscopic evolution of the tagged particle fluctuates around the solution of the ODE (1.1) and one usually writes

$$
\frac{d x}{d t}=b(x, t)+\frac{1}{\sqrt{L}} \xi(t)
$$

to model such fluctuations, where $\xi$ is the time derivative of some inhomogeneous diffusion.

If in SEP we assume that the particles jump as symmetric random walks, then the ODE vanishes $(\gamma=0)$ and so simplifies the fluctuation problem. In this case the problem was treated by Kipnis and Varadhan [6], assuming the model is in thermal equilibrium. The nonequilibrium case was studied in Quastel [9] and Rezakhanlou [11].

An important feature of ODE (1.1) is that for almost all $t$, a solution will avoid the discontinuity points of the density $\rho$, providing $\rho$ is positive at that point.

It is well known that if $\rho_{0}$ is in the class of functions of bounded variation, $B V$, then $\rho$ is also in $B V$ and therefore the discontinuity set of $\rho$ can be embedded in a countable collection of rectifiable curves. The discontinuity set of $\rho$ is called the shock set.

It is of interest to study the microscopic structure of the shock set. In [12] we derive an ODE of the form

$$
\frac{d y}{d t}=F^{\prime}(\rho(y, t))
$$

for the evaluation of a second class particle for some of the models discussed above. For the definition of the second class particles and related results, see chapter 6 of [16]. We will see in [12] that a Filippov solution to (1.7) follows either a characteristic line or a shock of (1.3).

The organization of this paper is as follows: Sect. 2 is devoted to the statement of our main results. In Sect. 3 and 4 an equation of the form (1.2) will be derived in connection with the two-dimensional marginals of $x(t)$. In Sect. 5 the uniqueness of (1.1) will be established. In the last section the proof of our main results will be presented. 


\section{Notation and Main Results}

The primary purpose of this section is the statement of the main results. We start with the definition of the so-called processus misanthrope that includes the SEP and ZRP.

Let $\left(p(z): z \in Z^{d}\right)$ be a probability density function of finite range. That is,

$$
\begin{cases}\sum_{z} p(z)=1, & p(z) \geqq 0, \\ p(z)=0, & \text { if }|z|>r,\end{cases}
$$

for some fixed $r$. We assume $p(z)+p(-z)$ is irreducible, i.e.

$$
\sum_{n>0}\left(p^{* n}(z)+p^{* n}(-z)\right)>0 \text {. }
$$

Let $b: \mathbb{N} \times \mathbb{N} \rightarrow[0, \infty)$ be a bounded function with the following properties:

(i) $b(0, \cdot) \equiv 0$

(ii) $n \mapsto b(n, m)$ is a nondecreasing function for each $m$

(iii) $m \mapsto b(n, m)$ is a nonincreasing function for each $n$.

Given $p$ and $b$ we define $\left(\eta_{t}(u): u \in \mathbb{Z}^{d}\right)$ as the unique Feller process with state space $E=\mathbb{N}^{\mathbb{Z}^{d}}$ and the infinitesimal generator $\mathscr{L}$, where $\mathscr{L}$ acting on cylinder functions, is defined by

where

$$
\mathscr{L} f(\eta)=\sum_{u, v} p(v-u) b(\eta(u), \eta(v))\left(f\left(\eta^{u v}\right)-f(\eta)\right),
$$

$$
\eta^{u v}(z)= \begin{cases}\eta(u)-1 & \text { if } z=u \\ \eta(v)+1 & \text { if } z=v \\ \eta(z) & z \neq u, v\end{cases}
$$

provided $\eta(u) \geqq 1$ and $u \neq v ; \eta^{u, v}=\eta$ otherwise.

Formula (2.2) says that at transition times of the Markov process $\eta_{t}$, a particle jumps from site $u$ to $v$ with a rate equal to $b(n, m)$, where $n$ is the number of particles at site $u$ and $m$ is the number of particles at site $v$. To study the evolution of a tagged particle, we assume that each particle at site $u$ jumps with the rate $\frac{b(n, m)}{n}$ so that the total rate of a jump from $u$ to $v$ is $b(n, m)$.

Since the Markov process $\eta$ keeps track of the occupation number of each site and not the location of each particle, it is necessary to define a new Markov process $\mathbf{x}$ that will provide us with the exact location of each particle in the system.

If the total number of particles in the configuration $\eta$ is finite,

$$
N=\sum_{u} \eta(u)<\infty,
$$

we label particles from 1 to $N$, and we write $x_{i}(t)$ for the location of the $i$ th particle at time $t$. Hence the state space is

$$
\hat{E}=\bigcup_{N=1}^{\infty}\left(\mathbb{Z}^{d}\right)^{N}
$$


We also define the transformation

$$
T_{i, z}: \widehat{E} \rightarrow \widehat{E}
$$

by $T_{i, z}\left(x_{1} \ldots x_{N}\right)=\left(y_{1} \ldots y_{N}\right)$, where $y_{i}=x_{i}+z$, and $y_{j}=x_{j}$ for $j \neq i$.

We define the Markov process $\mathbf{x}(t)$ as the Feller process with the infinitesimal generator

where

$$
\hat{\mathscr{L}} f(\mathbf{x})=\sum_{i, z} p(z) \frac{b\left(\eta\left(\mathbf{x} ; x_{i}\right), \eta\left(\mathbf{x} ; x_{i}+z\right)\right)}{\eta\left(\mathbf{x} ; x_{i}\right)}\left(f\left(\mathrm{~T}_{i, z} \mathbf{x}\right)-f(\mathbf{x})\right),
$$

$$
\eta(\mathbf{x} ; u)=\left\{\# i: x_{i}=u\right\}
$$

for every $\mathbf{x}=\left(x_{1} \ldots x_{N}\right) \in \hat{E}$, and $u \in \mathbb{Z}^{d}$.

It is not hard to see that $\eta$ defined by (2.4) is also a Markov process with the infinitesimal generator (2.2). To be more precise, if $f$ in (2.3) is merely a function of the occupation numbers $\eta$, then $\hat{\mathscr{L}} f=\mathscr{L} f$.

Some restrictions on $b$ are needed to ensure that the invariant measures of the process $\eta$ are of simple form. For example we formulate conditions that guarantee product measures are invariant.

Let $g: \mathbb{N} \rightarrow[0, \infty)$ be a given bounded nondecreasing function with $g(0)=0$. For such $g$, and any given $\lambda \in\left[0, \sup _{k} g(k)\right)$ we define a probability measure $\Theta_{\lambda}$ on $\mathbb{N}$ by

$$
\Theta_{\lambda}(n)= \begin{cases}\frac{1}{Z(\lambda)} \frac{\lambda^{n}}{g(1) \ldots g(n)} & \text { if } n \neq 0 \\ \frac{1}{Z(\lambda)} & \text { if } n=0,\end{cases}
$$

where $Z(\lambda)$ is the normalizing factor. Set

Then

$$
\varphi(\lambda)=\sum_{n=1}^{\infty} n \Theta_{\lambda}(n)
$$

$$
\varphi:\left[0, \sup _{k} g(k)\right) \rightarrow[0, \infty)
$$

is strictly increasing, and

$$
\lim _{\lambda \rightarrow \sup _{k} g(k)} \varphi(\lambda)=+\infty .
$$

The inverse of $\varphi$ is denoted by $\lambda(\cdot)$ and let $\Theta^{\rho}=\Theta_{\lambda(\rho)}$. The probability measure $v^{\rho}$ is obtained by taking the product of $\Theta^{\rho}$,

$$
v^{\rho}(d \eta)=\prod_{u \in \mathbb{Z}^{d}} \Theta^{\rho}(d \eta(u))
$$

so that $v^{\rho}(\eta(u)=k)=\Theta^{\rho}(k)$. We certainly have

$$
\begin{aligned}
\int \eta(0) v^{\rho}(d \eta) & =\rho, \\
\int g(\eta(0)) v^{\rho}(d \eta) & =\lambda(\rho) .
\end{aligned}
$$

For a given $b$ as in (2.1), we assume that there exists a bounded nondecreasing $g$ such that

$$
b(n, m-1) g(m)=b(m, n-1) g(n)
$$


for $n, m \geqq 1$. We also assume

$$
b(n, m)-b(m, n)=b(n, 0)-b(m, 0)
$$

for $n, m \geqq 0$. The conditions (2.7) and (2.8) imply $\left\{\nu^{\rho}: 0 \leqq \rho<\infty\right\}$ are invariant with respect to $\mathscr{L}$.

If we choose $b(n, m)=g(n)$ we obtain the ZRP. In this case the rate of the jump from $u$ to $v$ does not depend on the occupation number $\eta(v)$. Note that if $b(n, m)=g(n)$ both $(2.7)$ and $(2.8)$ are satisfied.

If we choose

$$
b(n, m)= \begin{cases}1 & \text { if } n=1, m=0 \\ 0 & \text { otherwise }\end{cases}
$$

and restricting the process $\eta$ to $\{0,1\} \mathbb{Z}^{d}$, we obtain the SEP. In this case there is at most one particle per site. We also restrict the $\mathbf{x}$-process to the set of configurations $\mathbf{x}=\left(x_{1} \ldots x_{N}\right)$ with $x_{i} \neq x_{j}$ if $i \neq j$. The rate function in (2.3) can be written as

$$
\frac{b\left(\eta\left(\mathbf{x} ; x_{i}\right), \eta\left(\mathbf{x} ; x_{i}+z\right)\right)}{\eta\left(\mathbf{x} ; x_{i}\right)}=1-\eta\left(\mathbf{x} ; x_{i}+z\right) \text {. }
$$

For the SEP, the invariant measures are $\left\{v^{\rho}: \rho \in[0,1]\right\}$, where each $v^{\rho}$ is a product measure with marginals $\Theta^{\rho}$, where

$$
\Theta^{\rho}(k)= \begin{cases}\rho & \text { if } k=1 \\ 1-\rho & \text { if } k=0\end{cases}
$$

We now describe the type of initial distribution that we will consider for the process $\mathbf{x}(t)$.

Notation 2.1 Let $\mu^{L}$ be a sequence of probability measures on $\hat{E}$ and let $\rho_{0}: \mathbb{R}^{d} \rightarrow[0, \infty)$ be a bounded integrable function. We then write $\mu^{L} \sim \rho_{0}$ if the following conditions hold:

(a) $\mu^{L}$ is symmetric. For each $N$ and every permutation $\sigma$

$$
\mu^{L}\left\{\left(x_{1} \ldots x_{N}\right)\right\}=\mu^{L}\left\{\left(x_{\sigma_{1}} \ldots x_{\sigma_{N}}\right)\right\} .
$$

(b) $\mu^{L}$ is a product measure.

(c) There exists a sequence $\rho_{u, L}$ such that

$$
\begin{gathered}
\mu^{L}(\eta(\mathbf{x} ; u)=k)=\Theta^{\rho_{u, L}}(k), \quad u \in \mathbb{Z}^{d}, k \in \mathbb{N}, \\
\lim _{L \rightarrow \infty} \int_{|x| \leqq l}\left|\rho_{[x L], L}-\rho_{0}(x)\right| d x=0
\end{gathered}
$$

for every positive $l$. ( $[x L]$ denotes the integer part of $x L$.)

Note that if $\rho_{0}$ is continuous, we may choose $\rho_{u, L}=\rho_{0}\left(\frac{u}{L}\right)$ for all $u \in \mathbb{Z}^{d}$.
We define the constant vector $\gamma$ as the mean of $p$,

$$
\gamma=\sum_{z} z p(z)
$$

and the scalar valued function $h$ as the average of $b$ with respect to the invariant measure $v^{\rho}$;

$$
h(\rho)=\int b(\eta(u), \eta(v)) v^{\rho}(d \eta), \quad u \neq v .
$$


Since $v^{\rho}$ is a translation invariant product measure, the right-hand side of (2.9) is independent of $u$ and $v$.

In the case of SEP, we have $h(\rho)=\rho(1-\rho)$, and in the case of ZRP we have $h(\rho)=\lambda(\rho)$.

Let $\rho(x, t)$ denote the unique entropy solution of

$$
\left\{\begin{array}{l}
\frac{\partial \rho}{\partial t}+\gamma \cdot \nabla h(\rho)=0 \\
\rho(x, 0)=\rho_{0}(x)
\end{array} .\right.
$$

Here the initial condition is understood in the following sense: for each $l$,

$$
\lim _{t \rightarrow 0|x| \leqq l}\left|\rho(x, t)-\rho_{0}(x)\right| d x=0 \text {. }
$$

A bounded integrable function $\rho$ is an entropy solution of (2.10) if (1.5) holds for every entropy pair $(\phi, q)$ with $F$ given by $F(\rho)=\gamma h(\rho)$.

Let $P^{L}$ denote the distribution of the process $\mathbf{x}(t)$ with the initial distribution $\mu^{L}$. In $[10]$ we proved

Theorem 2.2. For every bounded continuous function $J$, and every $t \geqq 0$,

$$
\lim _{L \rightarrow \infty} \int\left|\frac{1}{L^{d}} \sum_{i=1}^{N} J\left(\frac{1}{L} x_{i}(t L)\right)-\int J(x) \rho(x, t) d x\right| P^{L}(d \mathbf{x})=0 .
$$

It follows from our assumptions on the initial distributions $\mu_{L}$ that (2.11) holds initially with $\rho(x, t)$ replaced with $\rho_{0}(x)$. Thus Theorem 2.2 asserts that $(2.11)$ also holds at later macroscopic times with the density profile $\rho(x, t)$ that is the unique entropy solution of (2.10).

Note that the symmetry property of $\mu^{L}$ (part (a) in Notation 2.1 ) is redundant for Theorem 2.2 because the expression

$$
\frac{1}{L^{d}} \sum_{i=1}^{N} J\left(\frac{1}{L} x_{i}(t L)\right)
$$

is already symmetric. The symmetry property will however play an important role for the proof of Theorem 2.7 below, that concerns the macroscopic behavior of a tagged particle $x_{i}(t)$.

Let $\psi(\rho)$ be defined by

$$
\psi(\rho)=\left\{\begin{array}{cc}
\frac{h(\rho)}{\rho} & \text { if } \rho \neq 0 \\
h^{\prime}(0) & \text { if } \rho=0 .
\end{array}\right.
$$

Since $h$ is smooth, it is not hard to see that $\psi$ is also smooth. Define

$$
b(x, t)=\psi(\rho(x, t)) .
$$

Throughout this paper we assume any of the following assumptions:

Assumption 2.3. The initial density $\rho_{0}$ is of bounded variation in the direction $\gamma$ :

$$
\operatorname{Var}_{\gamma}\left(\rho_{0}\right)=\sup _{|r|>0} \int \frac{\left|\rho_{0}(x+r \gamma)-\rho_{0}(x)\right|}{|r|} d x<\infty .
$$


Assumption 2.4. $h$ is uniformly convex. That is, there exists a $\delta>0$ such that $h^{\prime \prime}(\rho) \geqq \delta$ for all $\rho$.

It is known that Assumption 2.3 implies $\rho$, as a function of $(x, t)$, is also of bounded $\gamma$-variation. Indeed

$$
\sup _{|r|>0, s>-t} \int \frac{|\rho(x+r \gamma, t+s)-\rho(x, t)|}{|r|+s} d x<\operatorname{Var} \rho_{0} .
$$

If (2.14) holds, we say $\rho$ is in $B \gamma V$. If, for example, $\gamma=(1,0, \ldots, 0),(2.14)$ implies that $\rho$, as a function of $x^{1}$ and $t$, is of bounded variation.

It is also known that under Assumption 2.3, there exists a constant $C=C(\delta)$ such that

$$
\rho(x+r \gamma, t)-\rho(x, t) \leqq C\left(1+\frac{1}{t}\right) r
$$

for every $x=\left(x^{1} \ldots x^{d}\right) \in \mathbb{R}^{d}$, and every $r, t>0$.

See Smoller [15] for (2.14) and (2.15). Let us mention that (2.14) and (2.15) are stated in [15] under the assumption $d=1$. To establish (2.15) in our case, we note that $\gamma$, the only nonscalar part of the flux, is constant. So, for example, if $\gamma=(1,0, \ldots, 0)$, is the unit vector parallel to the $x^{1}$-axis, one can solve $(2.10)$ by fixing $x^{2} \ldots x^{d}$, and solving it for $x^{1}$.

The main object of this article is to study the asymptotic behavior of $\frac{1}{L} x_{i}(t L)$ as $L$ goes to infinity. We claim that the limiting behavior of each particle is governed by the evolution of (1.1) where the initial condition is random and $b$ is given by (2.13).

As we mentioned earlier $b$ is discontinuous and we interpret (1.1) in the Filippov sense. Define

$$
(\text { ess } \lim \sup b)(z)=\lim _{\delta \rightarrow 0} \inf _{|M|=0} \sup _{z^{\prime} \in B_{\delta}(z)-M} b\left(z^{\prime}\right),
$$

where $|\cdot|$ denotes the Lebesgue measure, the infinum is over all sets of zero Lebesgue measure, and $B_{\delta}(z)$ denotes the ball of radius $\delta$ an center $z=(x, t)$. Similarly we define

$$
(\text { ess } \lim \inf b)(z)=(\text { ess } \lim \sup (-b))(z) .
$$

Definition 2.5 A Lipschitz function $x:[0, T] \rightarrow \mathbb{R}^{d}$ is a solution to (1.1) in Filippov sense if for almost all $t$,

$$
\frac{d x}{d t} \in[(\text { ess } \lim \inf b)(x, t),(\text { ess } \lim \sup b)(x, t)] .
$$

Under Assumption 2.3, $\rho$ is in $B \gamma V$, therefore $b$ is in $B \gamma V$. In particular, if $d=1$, one can modify $b$ on a set of zero Lebesgue measure such that

$$
b^{+}(x, t)=\lim _{y \downarrow x} b(y, t), \quad b^{-}(x, t)=\lim _{y \uparrow x} b(y, t)
$$

exist for every $x, t$. Similarly we define $\rho^{+}$and $\rho^{-}$. Hence, one can replace (2.16) with

$$
\frac{d x}{d t} \in\left[b^{+} \wedge b^{-}(x, t), b^{+} \vee b^{-}(x, t)\right],
$$

where $b^{+} \wedge b^{-}$denotes the minimum and $b^{+} \vee b^{-}$denotes the maximum of $b^{+}$ and $b^{-}$.

Our first theorem treats the uniqueness problem for Eq. (1.1). 
Theorem 2.6. There exists at most one solution $x(t)$ of the initial value problem

$$
\left\{\begin{array}{l}
\frac{d x}{d t}=b(x, t) \\
x(0, a)=a .
\end{array}\right.
$$

Moreover for almost all $t$, either $\rho^{+}(x(t), t)=\rho^{-}(x(t), t)$, or $\rho^{+}(x(t), t) \neq$ $\rho^{-}(x(t), t)$ and $\rho^{+}(x(t), t) \rho^{-}(x(t), t)=0$.

The proof of this theorem will be given in Sect. 5 .

Since $b$ is a product of a constant vector and a scalar function, it suffices to prove Theorem 2.6 under the assumption $d=1$.

Let $x(t, a)$ denote the unique solution of $(2.18)$. Let $\mathscr{D}=\mathscr{D}\left([0, \infty), \mathbb{R}^{d}\right)$ denote the space of right continuous functions with left limit that have values in $\mathbb{R}^{d}$. $\mathscr{D}$ is endowed with the skorohod topology, and as before $P^{L}$ is the distribution of the process $x(\cdot)$. Let

$$
y_{i, L}(t)=\frac{1}{L} x_{i}(t L)
$$

and let the probability $Q^{L}$ denote the distribution of $y_{i, L}$ 's. We view $Q^{L}$ as a probability measure defined on $\mathscr{D}$.

Let $R$ denote the probability law of $x(\cdot, a)$ with $a$ distributed according to

$$
R(a \in A)=\int_{A} c \rho_{0}(x) d x,
$$

where $c$ is the normalizing constant

$$
c^{-1}=\int_{\mathbb{R}^{d}} \rho_{0}(x) d x .
$$

The main result of this paper identifies $R$ as the asymptotic law of the sequence $Q^{L}$.

Theorem 2.7. Under Assumption 2.3, we have

$$
\lim _{L \rightarrow \infty} \int\left|\frac{1}{N} \sum_{i=1}^{N} J\left(y_{i}\right)-\int J d R\right| Q^{L}(d y)=0
$$

for every continuous function $J: \mathscr{D} \rightarrow \mathbb{R}$.

The proof of this theorem will be given in the last section.

In the language of [17], Theorem 2.7 says that the sequence $Q^{L}$ is $R$-chaotic.

Let $\mathscr{M}(\mathscr{D})$ denote the space of probability measures on $\mathscr{D}$. One can regard $\bar{X}_{N}=\frac{1}{N} \Sigma \delta_{y i, L}$ as a sequence of $\mathscr{M}(\mathscr{D})$-valued random variables, and (2.21) asserts that this sequence converges in law to a constant random variable $R$.

Indeed it is known that a statement like (2.21) is equivalent to the following ([17], chapter 1 ): if $J_{1} \ldots J_{k}: \mathscr{D} \rightarrow \mathbb{R}$ are $k$ continuous functions then

$$
\lim _{N \rightarrow \infty} \int J_{1}\left(y_{1}\right) \ldots J_{k}\left(y_{k}\right) Q^{L}(d y)=\prod_{i=1}^{k} \int J_{i}(y) R(d y) .
$$

As we mentioned earlier, the symmetry of $\mu^{L}$ plays an essential role in the proof of the previous theorem. In the case of SEP with $d=1$, and if only the nearest neighbor jumps are allowed, the symmetry assumption can be relaxed. 
Assumption 2.8. $d=1$, and $p(1)+p(-1)=1$.

Let $Q^{L}$ be as in Theorem 2.7, and let $Q_{a}^{L}$ denote the measure $Q^{L}$ conditioned such that the first particle starts from the site $[a L]$ :

$$
Q_{a}^{L}(d \mathbf{x})=Q^{L}\left(d \mathbf{x} \mid x_{1}(0)=[a L]\right) .
$$

Theorem 2.9. In the case of SEP and under Assumptions 2.8 and 2.3, we have

$$
\lim _{L \rightarrow \infty} \int\left|\frac{1}{L} x_{1}(t L)-x(t, a)\right| Q_{a}^{L}(d \mathbf{x})=0
$$

providing

for every $\varepsilon>0$.

$$
\int_{a}^{a \pm \varepsilon} \rho_{0}(x) d x \neq 0
$$

Theorem 2.9 and a variant of Theorem 2.7 (under Asumption 2.4) will be established in Sect. 6.

\section{Hydrodynamic Equation for the two color problem}

To analyze the two-dimensional density of $x_{i}$, we consider

$$
\frac{1}{N} \sum_{i=1}^{N} J_{1}\left(\frac{1}{L} x_{i}\left(t_{1} L\right)\right) J_{2}\left(\frac{1}{L} x_{i}\left(t_{2} L\right)\right),
$$

where $J_{1}, J_{2}$ are two measurable functions. We may choose $J_{1}(x)=\mathbb{1}_{A}(x)$, where $A$ is a measurable subset of $\mathbb{R}^{d}$, and $J_{2}$ a smooth function of compact support. For ease in notation let $y_{i, L}(t)=\frac{1}{L} x_{i}(t L)$, and suppose $t_{2}>t_{1}$. We can write (3.1) as

$$
\frac{1}{N} \Sigma^{\prime} J_{2}\left(y_{i, L}\left(t_{2}\right)\right)
$$

where the sum is now over $i$ for which $y_{i, L}\left(t_{1}\right) \in A$. We paint the $i^{\text {th }}$ particle blue if $y_{i, L}\left(t_{1}\right) \in A$ and we paint the remaining particles white. If we denote the density of the blue particles by $m(x, t)$, we have

$$
m\left(x, t_{1}\right)=\rho\left(x, t_{1}\right) \mathbb{1}_{A}(x)
$$

and $\rho(x, t)-m(x, t)$ would be the macroscopic density of the white particles. Let $\zeta_{t}$, $\xi_{t}$ denote the occupation number of blue and white particles respectively. It is not hard to see that $(\zeta, \xi)$ is also Markov process with state space

$$
\Omega=\mathbb{N}^{\mathbb{Z}^{d}} \times \mathbb{N}^{\mathbb{Z}^{d}}
$$

and the infinitesimal generator

$$
\begin{aligned}
\mathscr{A} f(\zeta, \xi)= & \sum_{u, v} p(v-u) \frac{\zeta(u)}{\eta(u)} b(\eta(u), \eta(v))\left(f\left(\zeta^{u v}, \xi\right)-f(\zeta, \xi)\right) \\
& +\sum_{u, v} p(v-u) \frac{\xi(u)}{\eta(u)} b(\eta(u), \eta(v))\left(f\left(\zeta, \xi^{u v}\right)-f(\zeta, \xi)\right) .
\end{aligned}
$$

The main object of this section is to derive a hydrodynamic equation for the density $m(x, t)$. 
To prepare for this, we start with some definitions. Let $\alpha^{L}(t, d x)=\frac{1}{L^{d}} \sum_{u} \zeta_{t L}(u)$ $\delta \frac{u}{L}(d x)$, i.e. if $J$ is a smooth function

$$
\int J(x) \alpha^{L}(t, d x)=\frac{1}{L^{d}} \sum_{u} J\left(\frac{u}{L}\right) \zeta_{t L}(u) .
$$

Initially the process $\left(\eta_{t}, \zeta_{t}\right)$ is distributed according to the probability measure $\gamma_{L}(d \eta, d \zeta)$ such that the $\eta$-marginal of $\gamma_{L}$ is a product measure as in Notation 2.1 while the $\zeta$-marginal is chosen such that for every smooth function $J$,

$$
\lim _{L \rightarrow \infty} \int\left|\frac{1}{L^{d}} \sum_{u} J\left(\frac{u}{L}\right) \zeta(u)-\int J(x) m_{0}(x) d x\right| \gamma_{L}(d \eta, d \zeta)=0
$$

for some measurable function $m_{0}(x) \leqq \rho_{0}(x)$. Hence $m_{0}(x)$ represents the macroscopic density of the blue particles at time $t=0$. Let $S^{L}$ be the law of $\alpha^{L} ; S^{L}$ is a probability measure on the Skorohod space $D\left([0, \infty) ; \mathscr{M}\left(\mathbb{R}^{d}\right)\right.$ where $\mathscr{M}\left(\mathbb{R}^{d}\right)$ denotes the space of Radon measures on $\mathbb{R}^{d}$. We will show

Theorem 3.1. (a) $\left\{S^{L}\right\}$ is tight. (b) If $S$ is any limit point of $\left\{S^{L}\right\}$ then $S$ is concentrated on the set of measures $\alpha(t, d x)$ such that

$$
\alpha(t, d x) \ll d x
$$

and if $\alpha(t, d x)=m(x, t) d x, m(x, t)$ satisfies

$$
\left\{\begin{array}{l}
\frac{\partial}{\partial t} m(x, t)+\operatorname{div}(b(x, t) m(x, t))=0 \\
m(x, 0)=m_{0}(x)
\end{array}\right.
$$

where $b$ is defined by (2.13).

The proof of this theorem will be given at the end of this section.

In the case of SEP, the Bernoulli measures are invariant. More precisely, for every pair $\left(\rho_{1}, \rho_{2}\right)$ with $\rho_{1}, \rho_{2}, \rho_{1}+\rho_{2} \in[0,1]$, define the product measure $v^{\rho_{1}, \rho_{2}}$ such that

$$
\begin{gathered}
v^{\rho_{1}, \rho_{2}}(\zeta(u)=1)=\rho_{1}, \\
v^{\rho_{1}, \rho_{2}}(\xi(u)=1)=\rho_{2} .
\end{gathered}
$$

Then the measure $v^{\rho_{1}, \rho_{2}}$ is invariant for $\mathscr{A}$.

In general, for every pair $\left(\lambda_{1}, \lambda_{2}\right)$, we define

$$
\Theta_{\lambda_{1}, \lambda_{2}}\left(k_{1}, k_{2}\right)=\frac{1}{Z\left(\lambda_{1}, \lambda_{2}\right)} \frac{\lambda_{1}^{k_{1}} \lambda_{2}^{k_{2}}}{g(1) \ldots g(k)} \frac{k !}{k_{1} ! k_{2}},
$$

where $k=k_{1}+k_{2}$ and $Z\left(\lambda_{1}, \lambda_{2}\right)$ is the normalizing factor. $\Theta$ is a probability measure on $\mathbb{N} \times \mathbb{N}$.

$$
\begin{aligned}
& \rho_{1}=\sum_{k_{1}, k_{2}} k_{1} \Theta_{\lambda_{1}, \lambda_{2}}\left(k_{1}, k_{2}\right), \text { and } \\
& \rho_{2}=\sum_{k_{1}, k_{2}} k_{2} \Theta_{\lambda_{1}, \lambda_{2}}\left(k_{1}, k_{2}\right) .
\end{aligned}
$$

First we observe

$$
Z\left(\lambda_{1}, \lambda_{2}\right)=\sum_{k_{1}, k_{2}} \frac{\lambda_{1}^{k_{1}} \lambda_{2}^{k_{2}}}{g(1) \ldots g(k)} \frac{k !}{k_{1} ! k_{2} !}=Z\left(\lambda_{1}+\lambda_{2}\right) .
$$


We certainly have

$$
\begin{gathered}
\lambda_{1} \frac{d Z}{d \lambda_{1}}=\rho_{1} Z, \\
\left(\lambda_{1}+\lambda_{2}\right) \frac{d Z}{d \lambda_{1}}=\left(\rho_{1}+\rho_{2}\right) Z .
\end{gathered}
$$

Here $Z=Z\left(\lambda_{1}, \lambda_{2}\right)$. Therefore

$$
\lambda_{1}=\frac{\rho_{1}}{\rho} \lambda(\rho)
$$

where $\rho=\rho_{1}+\rho_{2}$. We now define

$$
\Theta^{\rho_{1}, \rho_{2}}=\Theta_{i_{1}, \lambda_{2}}
$$

with $\lambda_{1}=\frac{\rho_{1}}{\rho} \lambda(\rho)$ and $\lambda_{2}=\frac{\rho_{2}}{\rho} \lambda(\rho)$. We also take $v^{\rho_{1}, \rho_{2}}$ to be the product of $\Theta^{\rho_{1}, \rho_{2}}$ :

$$
v^{\rho_{1}, \rho_{2}}\left(\zeta(u)=k_{1}, \zeta(u)=k_{2}\right)=\Theta^{\rho_{1}, \rho_{2}}\left(k_{1}, k_{2}\right) .
$$

It is not hard to see that $v^{\rho_{1}, \rho_{2}}$ are invariant with respect to $\mathscr{A}$.

The main ingredients for the proof of Theorem 3.1 are the following lemmas.

Lemma 3.2. For every $u, v$ with $u \neq v$, we have

$$
\int \frac{\zeta(u)}{\eta(u)} b(\eta(u), \eta(v)) v^{\rho_{1}, \rho_{2}}(d \zeta, d \xi)=\frac{h(\rho)}{\rho} \rho_{1} .
$$

Proof. Set $\zeta(u)=n_{1}, \xi(u)=n_{2}, \zeta(v)=m_{1}, \xi(v)=m_{2}, n_{1}+n_{2}=n, m_{1}+m_{2}=m, \lambda_{1}=$ $\lambda_{1}\left(\rho_{1}, \rho_{2}\right), \lambda_{2}=\lambda_{2}\left(\rho_{1}, \rho_{2}\right)$. Then the left-hand side of (3.4) equals to

$$
\frac{1}{Z^{2}\left(\lambda_{1}, \lambda_{2}\right)} \sum \frac{n_{1}}{n} b(n, m) \frac{\lambda_{1}^{n_{1}} \lambda_{2}^{n_{2}}}{g(1) \ldots g(n)} \frac{n !}{n_{1} ! n_{2} ! g(1) \ldots g(m)} \frac{\lambda_{1}^{m_{1}} \lambda_{2}^{m_{2}}}{m_{1} ! m_{2} !},
$$

where the sum is over $n_{1}, n_{2}, m_{1}$ and $m_{2}$. On the other hand

$$
\sum_{n_{1}+n_{2}=n} \frac{n_{1}}{n} \lambda_{1}^{n_{1}} \lambda_{2}^{n_{2}} \frac{n !}{n_{1} ! n_{2} !}=\lambda_{1}\left(\lambda_{1}+\lambda_{2}\right)^{n-1},
$$

which follows from the identity

$$
\left(\lambda_{1}+\lambda_{2}\right)^{n}=\sum_{n_{1}+n_{2}=n} \lambda_{1}^{n_{1}} \lambda_{2}^{n_{2}} \frac{n !}{n_{1} ! n_{2} !}
$$

after differentiating with respect to $\lambda_{1}$. Therefore the left-hand side of (3.4) equals to

$$
\frac{1}{Z^{2}\left(\lambda_{1}, \lambda_{2}\right)} \sum b(n, m) \frac{\left(\lambda_{1}+\lambda_{2}\right)^{n-1}}{g(1) \ldots g(n)} \frac{\lambda_{1}^{m_{1}} \lambda_{2}^{m_{2}}}{g(1) \ldots g(m)} \frac{m !}{m_{1} ! m_{2} !} \lambda_{1} .
$$


Using (3.5) with $n$ replaced with $m$, and using $Z\left(\lambda_{1}, \lambda_{2}\right)=Z\left(\lambda_{1}+\lambda_{2}\right)$, we can write that the sum equals to

$$
\begin{aligned}
& \frac{1}{Z^{2}\left(\lambda_{1}+\lambda_{2}\right)} \sum b(n, m) \frac{\left(\lambda_{1}+\lambda_{2}\right)^{n}}{g(1) \ldots g(n)} \frac{\left(\lambda_{1}+\lambda_{2}\right)^{m}}{g(1) \ldots g(m)} \frac{\lambda_{1}}{\lambda_{1}+\lambda_{2}} \\
& =\frac{\lambda_{1}}{\lambda_{1}+\lambda_{2}} \int b(\eta(u), \eta(v)) v \rho(d \eta) \\
& =\frac{\lambda_{1}(\rho)}{\lambda(\rho)} h(\rho)
\end{aligned}
$$

which equals to $\frac{\rho_{1}}{\rho} h(\rho)$ by (3.3).

Lemma 3.3 (One block estimates). Let $\gamma^{L}$ be a sequence of probability measures satisfying the properties mentioned in the beginning of this section. Then for every local function $f$ (depending on finitely many coordinates), every test function $J$ of compact support and every $t_{0}>0$, we have

$$
\begin{aligned}
\lim _{l \rightarrow \infty} \lim _{L \rightarrow \infty} \int_{0}^{t_{0}} \int \mid \frac{1}{L^{d}} \sum_{u} J\left(\frac{u}{L}\right) \tau_{u} f\left(\zeta_{t L}, \xi_{t L}\right)- \\
\quad \frac{1}{L^{d}} \sum_{u} J\left(\frac{u}{L}\right) \hat{f}\left(\frac{1}{\left|T_{l}(u)\right|} \sum_{v \in T_{l}(u)} \zeta(v), \frac{1}{\left|T_{l}(u)\right|} \sum_{v \in T_{l}(u)} \xi(v)\right) \mid \gamma^{L}(d \zeta, d \xi) d t=0,
\end{aligned}
$$

where $\hat{f}\left(\rho_{1}, \rho_{2}\right)=\int f(\zeta, \xi) v^{\rho_{1}, \rho_{2}}(d \zeta, d \xi), T_{l}(u)$ is the d-dimensional cube in $\mathbb{Z}^{d}$ with center $u$, side length $l$, and volume $\left|T_{l}(u)\right|$, and $\tau_{u}$ denotes the space-shift.

The proof of this lemma is the content of the next section.

While Lemma 3.3 establishes some type of law of large numbers for the $(\zeta, \xi)$ configurations at the microscopic level, the next lemma deals with the fluctuations of the microscopic densities $\frac{1}{\left|T_{l}(u)\right|} \sum_{v \in T_{l}(u)} \eta_{t L}(v)$ on a macroscopic scale.

Associated with a configuration $\eta_{t L}$, we define the Young measure $\pi^{L, l}$ by

$$
\int \Phi(x, \lambda) \pi^{L, l}(t, d x ; d \lambda)=\frac{1}{L^{d}} \sum_{u} \Phi\left(\frac{u}{L}, M_{T_{l}(u)}\left(\eta_{t L}\right)\right) .
$$

The map $\eta_{t L} \mapsto\left(\pi^{L, l}\right)$ induces a probability measure $\mathscr{R}^{L, l}$ on $X$, where $X$ is the space of measurable maps from $[0, \infty)$ into the space of Radon measures on $\mathbb{R}^{d} \times \mathbb{R}^{+}$. It is not hard to see that the sequence $\left\{\mathscr{R}^{L, l}\right\}$ is tight as a sequence of probability measures on $X$. First we let $L$ go to infinity. Let $\left\{\mathscr{R}^{l}\right\}$ be a sequence of limit points. Then we let $l$ go to infinity. Let $\mathscr{R}$ be any limit point. The following lemma can be found in Sect. 5 of [10].

Lemma 3.4. For almost all $\pi$ with respect to $\mathscr{R}$, we have

$$
\int \Phi(x, \lambda) \pi(t, d x ; d \lambda)=\int \Phi(x, \rho(x, t)) d x,
$$

where $\rho$ is the unique entropy solution of (2.10), and $\Phi$ is any continuous function.

Symbolically this can be written as

$$
\pi(t, d x ; d \lambda)=\delta_{\rho(x, t)}(d \lambda) d x .
$$


Proof of Theorem 3.1. (a) Let $J$ be a smooth test function. It is not hard to see

$$
\begin{aligned}
\frac{1}{L^{d}} \sum_{u} J\left(\frac{u}{L}\right) \zeta_{t L}(u)= & \frac{1}{L^{d}} \sum_{u} J\left(\frac{u}{L}\right) \zeta_{0}(u) \\
& +\frac{1}{L^{d}} \int_{0}^{t L} \sum_{u, z} p(z) \frac{\zeta_{s}(u)}{\eta_{s}(u)} b\left(\eta_{s}(u), \eta_{s}(v)\right)\left(J\left(\frac{u+z}{L}\right)-J\left(\frac{u}{L}\right)\right) \\
& +M_{t L},
\end{aligned}
$$

where $M_{t L}$ is a martingale with the quadratic variation

$$
\begin{aligned}
E M_{t L}^{2} & =\frac{1}{L^{2 d}} \int_{0}^{t L} \sum_{u, z} p(z) \frac{\zeta_{s}(u)}{\eta_{s}(u)} b\left(\eta_{s}(u), \eta_{s}(v)\right)\left(J\left(\frac{u+z}{L}\right)-J\left(\frac{u}{L}\right)\right)^{2} \\
& =O\left(\frac{1}{L^{d+1}}\right) .
\end{aligned}
$$

By Doob's inequality

$$
E \sup _{0 \leqq t \leqq t_{0}} M_{t L}^{2} \leqq \frac{\text { const. }}{L^{d+1}}
$$

for every fixed $t_{0}$. This and (3.7) implies

$$
\lim _{\delta \rightarrow 0} \varlimsup_{L \rightarrow \infty} P^{L}\left(\sup _{\substack{|t-s|<\delta \\ 0 \leqq t, s \leqq t_{0}}}\left|\frac{1}{L^{d}} \sum_{u} J\left(\frac{u}{L}\right)\left(\zeta_{t L}(u)-\zeta_{s L}(u)\right)\right|>\varepsilon\right)=0
$$

for every positive $\varepsilon$. This implies the tightness of the sequence $Q^{L}$.

(b) The absolute continuity of $\alpha$ with respect to the Lebesgue measure follows from $\zeta \leqq \eta$. Indeed the measure

$$
\frac{1}{L^{d}} \sum_{u} \eta_{t L} \delta \frac{u}{L}
$$

converges to a measure that is absolutely continuous with respect to Lebesgue measure and its density is $\rho(x, t)$. Therefore

$$
\alpha(t, d x) \leqq \rho(t, x) d x
$$

and this implies $\alpha$ is absolutely continuous.

Now let $J:[0, \infty) \times \mathbb{R}^{d} \rightarrow \mathbb{R}$ be a smooth function with compact support. Set

$$
B(s, \zeta)=\frac{1}{L^{d}} \sum_{u} J\left(\frac{u}{L}, \frac{s}{L}\right) \zeta(u) .
$$

Since $J$ has compact support, we have

$$
\hat{M}_{t L}=\int_{0}^{t L}\left[\frac{\partial}{\partial s} B\left(s, \zeta_{s}\right)+(\mathscr{A} B)\left(s, \zeta_{s}, \zeta_{s}\right)\right] d s+B\left(0, \zeta_{0}\right)
$$

is a Martingale for large $t$, and its quadratic variation is

$$
\langle\hat{M}\rangle_{t L}=\int_{0}^{t L}\left(\mathscr{A} B^{2}-2 B \mathscr{A} B\right)\left(\eta_{s}, \zeta_{s}\right) d s
$$


As in part (a), we have

$$
\lim _{L \rightarrow \infty} E^{L} \sup _{0 \leqq t \leqq t_{0}} \hat{M}_{t L}^{2}=0
$$

for every $t_{0}$. On the other hand the right-hand side of (3.8) equals to

$$
\begin{aligned}
\int_{0}^{\infty} \frac{1}{L^{d}} & \sum_{u} \frac{\partial J}{\partial s}\left(s, \frac{u}{L}\right) \zeta_{s L}(u) d s \\
& +\int_{0}^{\infty} \frac{1}{L^{d}} \sum_{u, z} p(z) z \cdot \nabla J\left(s, \frac{u}{L}\right) \frac{\zeta_{s L}(u)}{\eta_{s L}(u)} b\left(\eta_{s L}(u), \eta_{s L}(u+z)\right) d s \\
& +\frac{1}{L^{d}} \sum_{u} J\left(0, \frac{u}{L}\right) \eta_{0}(u)+r_{L}
\end{aligned}
$$

with $r_{L}$ an error of order $O\left(\frac{1}{L}\right)$. We know from Lemma 3.2 that the local function

$$
\frac{\zeta(u)}{\eta(u)} b(\eta(u), \eta(u+z))
$$

in the second term can be replaced by its average with respect to the invariant measures. Note that (3.11) in the case of SEP would look like

$$
\zeta(u)(1-\eta(u+z)) \text {. }
$$

In any case the average of (3.11) with respect to the invariant measure with densities $\rho_{1}$ and $\rho_{2}=\rho-\rho_{1}$ equals to $\frac{h(\rho)}{\rho} \rho_{1}$. For simplicity we write $G\left(\rho, \rho_{1}\right)$ for $\frac{h(\rho)}{\rho} \rho_{1}$. Now we can write the second term in (3.11) as

$$
\int_{0}^{\infty} \frac{1}{L^{d}} \sum_{u, z} p(z) z \cdot \nabla J\left(s, \frac{u}{L}\right) \quad G\left(M_{T_{l}(u)}(\eta), M_{T_{l}(u)}(\zeta)\right)+r_{L, l}^{\prime},
$$

where $r_{L, l}^{\prime}$ goes to zero in probability if $L$ goes to infinity and then $l$ goes to infinity.

Our next goal is to replace $T_{l}(u)$ in (3.12) with $T_{L \varepsilon}(u)$.

First we define the Young measures $\pi^{L, l}\left(t, d x ; d \lambda, d \lambda_{1}\right)$ by

$$
\int \Phi\left(x, \lambda, \lambda_{1}\right) \pi^{L, l}\left(t, d x ; d \lambda, d \lambda_{1}\right)=\frac{1}{L^{d}} \sum_{u} \Phi\left(\frac{u}{L}, M_{T_{l}(u)}(\eta), M_{T_{l}(u)}(\zeta)\right)
$$

for every test function $\Phi$. The elementary inequality

$$
\int J(x) \Phi_{0}\left(\lambda, \lambda_{1}\right) \pi^{L, l}\left(t, d x ; d \lambda, d \lambda_{1}\right) \leqq\left\|\Phi_{0}\right\|_{\infty}\left(\frac{1}{L^{d}} \sum_{u} J\left(\frac{u}{L}\right)\right)
$$

implies that as $L \rightarrow \infty, l \rightarrow \infty$, the weak limits of the distributions of $\pi^{L, l}$ are concentrated on measures $\pi\left(t, d x ; d \lambda, d \lambda_{1}\right)$ of the form

$$
\pi\left(t, d x ; d \lambda, d \lambda_{1}\right)=\pi\left(t, x ; d \lambda, d \lambda_{1}\right) d x .
$$

On the other hand Lemma 3.3 implies for almost all $(t, x)$, the measure $\pi\left(t, x ; d \lambda, d \lambda_{1}\right)$ is concentrated on the set $\{\rho(x, t)\} \times[0, \infty)$. This immediately implies

$$
\int \frac{h(\lambda)}{\lambda} \lambda_{1} \pi\left(t, x ; d \lambda, d \lambda_{1}\right)=b(x, t) \int \lambda_{1} \pi\left(t, x ; d \lambda, d \lambda_{1}\right)=b(x, t) m(x, t) .
$$


This, (3.7) and (3.9) imply

$$
\lim _{\varepsilon \rightarrow 0} \limsup S_{L \rightarrow \infty}^{L}\{x:|v(L, \varepsilon ; J)|>\delta\}=0
$$

for every positive $\delta$, where

$$
\begin{aligned}
v(L, \varepsilon ; J)= & \int_{0}^{\infty} \int \frac{\partial J}{\partial s}(x, s) m(x, s) d s \\
& +\int_{0}^{\infty} \int \gamma \cdot \nabla J(x, s) b(x, t)\left(\frac{1}{\left|B_{\varepsilon}(x)\right|} \int_{B_{\varepsilon}(x)} m(y, t) d y\right) d x \\
& +\int J(x, 0) m(x, 0) d x
\end{aligned}
$$

and this completes the proof of (b).

\section{One Block Estimates}

In this section we shall prove Lemma 3.3. The idea of the proof is to show that the distribution of $\left(\zeta_{t L}, \xi_{t L}\right)$ is close to a mixture of invariant measures for large $L$. This can be done by showing that the entropy production of the process at time $t L$, divided by the number of sites $L^{d}$, goes to zero. In fact we will show that the entropy production per site is of order $\frac{1}{L}$. Although a bound of order $\frac{1}{L}$ is enough to prove Lemma 3.3, it is not good enough to prove something like Lemma 3.3 for the process $\zeta$. Fortunately our hydrodynamic equation $(2.10)$ is linear in " $m$ " and only nonlinear in $\rho$, so one block estimates for $(\zeta, \xi)$, and two block estimates for $\eta$ will provide us with all the necessary estimates.

The idea of the proof is not new. In the pioneering work of Guo et al [4], entropy techniques were used to prove results of the type Lemmas 3.2 and 3.3.

By finite speed of propagation, we only need to prove Lemma 3.3 in the periodic case (see for example Lemma 5.7 of [10]). In other words we assume $\gamma^{L}(d \eta, d \zeta)$ has a density $f_{L}(d \eta, d \zeta)$ with respect to $v^{\rho_{1}, \rho_{2}}(d \eta, d \zeta)$ that is periodic. For simplicity we assume that $f_{L}$ and $\nu^{\rho_{1}, \rho_{2}}$ are defined on the periodic lattice $S_{L}^{d}=$ $\left\{u=\left(u_{1} \ldots u_{d}\right) \in \mathbb{Z}^{d}: u_{i} \in S_{L}\right\}$, where $S_{L}$ is the interval $[0, L]$ with 0 and $L$ identified. We further assume that the entropy per site is bounded:

$$
\sup _{L} \frac{1}{L^{d}} \int f_{L} \log f_{L} d v_{L} \leqq C
$$

for some constant $C$. Here $v_{L}$ denotes the periodic invariant measure.

Let $f_{L}^{t}$ be the solution to the forward equation

$$
\partial_{t} f_{L}^{t}=\mathscr{A}^{*} f_{L}^{t}
$$

where $\mathscr{A}^{*}$ denotes the adjoint of $\mathscr{A}$ with respect to $v_{L}$. If initially $(\zeta, \xi)$ is distributed according to the probability measure $f_{L} d v_{L}$, then at later times it is distributed according to $f_{L}^{t} d v_{L}$. An easy computation leads to

$$
\partial_{t} \int f_{L}^{t} \log f_{L}^{t} d v_{L}=\int \log f_{L}^{t} \mathscr{A}^{*} f_{L}^{t} d v_{L}
$$

It is well known that the entropy is nonincreasing in time. Therefore

$$
-\int \log f \mathscr{A}^{*} f d v_{L} \geqq 0
$$


for every density $f$. Equation (4.3) and the entropy bound (4.1) imply

$$
-\frac{1}{L^{d}} \int_{0}^{t L} \int \log f_{L}^{s} \mathscr{A}^{*} f^{s} d v_{L} \leqq C .
$$

If $p_{1}(z)=\min (p(z), p(-z))$ is irreducible, one can prove Lemma 3.3 by standard arguments. More precisely, let us write $p(z)=p_{1}(z)+p_{2}(z)$ for some $p_{2}(z) \geqq 0$. Let $\mathscr{A}_{1}, \mathscr{A}_{2}$ denote the corresponding generators with $p(z)$ replaced with $p_{1}(z)$ and $p_{2}(z)$ respectively. Then (4.4) holds for $\mathscr{A}_{1}$, and $\mathscr{A}_{2}$. Thus (4.5) implies

$$
-\frac{1}{L^{d}} \int_{0}^{t L} \int \log f_{L}^{s} \mathscr{A}_{1}^{*} f^{s} d v_{L} \leqq C .
$$

Since $p_{1}$ is symmetric and irreducible, we can apply the arguments of [4] or [16] (chapter 3) to deduce Lemma 3.3.

The general case can be treated with the help of some large deviations type estimates.

Let $\Gamma_{L}\left(\hat{\Gamma}_{L}\right)$ denote the law of the process $(\zeta, \xi)$ such that initially it is distributed according to $v_{L}\left(\gamma_{L}\right)$. We certainly have

$$
\frac{d \hat{\Gamma}_{L}}{d \Gamma_{L}}(\zeta, \xi)=f_{L}\left(\zeta_{0}, \xi_{0}\right)
$$

where the left-hand side is the Radon-Nikodym derivative evaluated at $(\zeta, \xi)$, and the right side is a function of $\left(\zeta_{0}, \xi_{0}\right)$ only.

Let $F_{L}$ denote the integrand of (3.6). Then (3.6) can be written as

$$
\lim _{l \rightarrow \infty} \limsup \iint_{L}^{t_{0}} F_{L}\left(\zeta_{s L}, \xi_{s L}\right) d s d \Gamma_{L}(\zeta, \xi)=0
$$

Now using the entropy inequality and (4.6) we have

$$
\begin{aligned}
\iint_{0}^{t_{0}} F_{L}\left(\zeta_{s}, \xi_{s}\right) d s d \Gamma_{L}(\zeta, \xi) \leqq & \frac{1}{\alpha} \frac{1}{L^{d}} \int f_{L} \log f_{L} d v_{L} \\
& +\frac{1}{L^{d}} \log \int \exp \left(\alpha L^{d} \int_{0}^{t_{0}} F_{L}\left(\zeta_{s L}, \xi_{s L}\right) d s\right),
\end{aligned}
$$

for every positive $\alpha$. Therefore, it suffices to show

$$
\lim _{l \rightarrow \infty} \limsup _{L \rightarrow \infty} \frac{1}{L^{d}} \log \int \exp \left(\alpha L^{d} \int_{0}^{t_{0}} F_{L}\left(\zeta_{s L}, \xi_{s L}\right) d s\right) d \Gamma_{L}(\zeta, \xi)=0 .
$$

Let $\mathscr{S}=\frac{1}{2}\left(\mathscr{A}+\mathscr{A}^{*}\right)$. In Lemma 4.1 below we show that the left-hand side of (4.8) is bounded above by

$$
\lim _{l \rightarrow \infty} \limsup _{L \rightarrow \infty} \sup _{f}\left[\int \alpha F_{L} f d v_{L}-L \mathscr{E}_{L}(f)\right],
$$

where the supremum is over densities $f$ such that $\int f d v_{L}=1$, and

$$
\mathscr{E}_{L}(f)=-\int \sqrt{f} \mathscr{S}(\sqrt{f}) d v_{L}
$$

Now we apply the arguments of [16], chapter 3 to conclude (4.9) is zero for every $\alpha$ positive. 
Lemma 4.1. For every function $F$ and every $t>0$, we have

$$
\frac{1}{t} \log \int \exp \left(\int_{0}^{t} F\left(\zeta_{s}, \xi_{s}\right) d s\right) d \Gamma_{L}(\zeta, \xi) \leqq \sup _{f}\left[\int F f d v_{L}-\mathscr{E}_{L}(f)\right] .
$$

Proof. Let $\mathscr{A}_{F}$ denote the infinitesimal generator defined by

$$
\mathscr{A}_{F} u=\mathscr{A} u+F u,
$$

where $F u$ is the product of $F$ and $u$.

Let $T_{t}^{F}$ denote the semigroup generated by $\mathscr{A}_{F}$. Then

$$
\left(T_{t}^{F_{1}} \mathbb{1}\right)\left(\zeta_{0}, \xi_{0}\right)=E^{\left(\zeta_{0}, \xi_{0}\right)} e^{\int_{0}^{t} F\left(\zeta_{0}, \xi_{0}\right) d s},
$$

where $\mathbb{1}$ denotes the constant function and $E^{\left(\zeta_{0}, \xi_{0}\right)}$ denotes the expectation with respect to the distribution of $(\zeta, \xi)$, conditioned to start from $\left(\zeta_{0}, \xi_{0}\right)$ at time $t=0$. Let $u\left(\zeta_{0}, \xi_{0}, t\right)$ denote the left-hand side of (4.11). By semigroup theory

Therefore

$$
\partial_{t} u=\mathscr{A}_{F} u \text {. }
$$

$$
\begin{aligned}
\partial_{t} \int u^{2}\left(\zeta_{0}, \xi_{0}, t\right) d v_{L}\left(\zeta_{0}, \xi_{0}\right) & =2 \int u \mathscr{A} u d v_{L}+2 \int F u^{2} \\
& =2 \int u \mathscr{S} u d v_{L}+2 \int F u^{2} \\
& \leqq 2 \sup _{f}\left[\int F f d v_{L}-\mathscr{E}_{L}(f)\right] \cdot\left(\int u^{2} d v_{L}\right)
\end{aligned}
$$

Let $\lambda_{F}$ denote the term with the supremum. Hence

Thus

$$
\int u^{2} d v_{L} \leqq e^{2 \lambda_{F} t}
$$

$$
\int u d v_{L} \leqq e^{2 \lambda_{F} t}
$$

and this is simply (4.10).

\section{Uniqueness}

This section is devoted to the uniqueness question for the ODE

$$
\left\{\begin{array}{l}
\frac{d x}{d t}=b(x, t) \text { for } t>t_{0}, \text { and } \\
x\left(t_{0}\right)=x_{0}
\end{array}\right.
$$

and the PDE

$$
\left\{\begin{array}{l}
m_{t}+(b(x, t) m)_{x}=0 \text { for } t>t_{0}, \text { and } \\
m\left(x, t_{0}\right)=m_{0}(x)
\end{array},\right.
$$

where (5.1) is understood in Filippov sense and (5.2) is understood in the distributional sense.

As before

$$
b(x, t)=\psi(\rho(x, t))
$$


where $\rho$ is the unique entropy solution of

$$
\left\{\begin{array}{l}
\rho_{t}+(h(\rho))_{x}=0 \\
\rho(x, 0)=\rho_{0}(x)
\end{array},\right.
$$

where $\psi$ is given by (2.12), i.e. if $\rho \neq 0$,

$$
\psi(\rho)=\frac{h(\rho)}{\rho} .
$$

Here, for simplicity, we have assumed $\gamma=1$, and $d=1$. The general case can be treated analogously.

Throughout this section, we assume that $\rho$ is in the class $\mathrm{BV}$, and $\rho_{0} \in L^{1} \cap L^{\infty}$.

Our main lemma toward the uniqueness problem of both (5.1) and (5.2) asserts that if $x(t)$ is a solution to (5.1), and $m$ is a solution to (5.2), then

$$
\int_{-\infty}^{x(t)} m(y, t) d y=\int_{-\infty}^{x\left(t_{0}\right)} m\left(y, t_{0}\right) d y .
$$

It is well known that for a function in $\mathrm{BV}$, each point is either a point of approximate continuity or a point of approximate jump discontinuity, with the possible exception of a set of zero 1-dimensional Hausdorff measure.

At a point of approximate continuity, we have

$$
\lim _{\delta \rightarrow 0} \delta^{-2} \int_{B_{\delta}(x, t)}|\rho(y, s)-\rho(x, t)| d y d s=0,
$$

where $B_{\delta}(x, t)$ is a ball of radius $\delta$, centered at $(x, t)$. At the jump point there are two distinct values $\rho_{ \pm}(x, t)$ and a unique direction $v$ such that

$$
\lim _{\delta \rightarrow 0} \delta^{-2} \int_{B_{\delta}(x, t) \cap H_{v}^{ \pm}}\left|\rho(y, s)-\rho_{ \pm}(x, t)\right| d y d s=0,
$$

where

and

$$
H_{v}^{+}=\left\{z: z \in \mathbb{R}^{2},(z-(x, t)) \cdot v \geqq 0\right\}
$$

$$
H_{v}^{-}=\left\{z: z \in \mathbb{R}^{2},(z-(x, t)) \cdot v \leqq 0\right\} .
$$

See Zeimer [18] for a proof.

Let $b_{ \pm}(x, t)=\psi\left(\rho_{ \pm}(x, t)\right)$. After modifying $\rho$ on a set of zero Lebesgue measure, we can assume that for almost all $t$

$$
\begin{aligned}
& \rho^{+}(x, t)=\lim _{y \downarrow x} \rho(y, t), \quad \text { and } \\
& \rho^{-}(x, t)=\lim _{y \uparrow x} \rho(y, t)
\end{aligned}
$$

exist for all $x$. Note that here we used superscripts but in (5.7) we used subscripts. We define

$$
\begin{gathered}
U(x, t)=\max \left(\psi\left(\rho^{-}(x, t)\right), \psi\left(\rho^{+}(x, t)\right)\right), \\
L(x, t)=\min \left(\psi\left(\rho^{-}(x, t)\right), \psi\left(\rho^{+}(x, t)\right)\right), \\
u(x, t)=\max \left(\psi\left(\rho_{-}(x, t)\right), \psi\left(\rho_{+}(x, t)\right)\right), \\
l(x, t)=\min \left(\psi\left(\rho_{-}(x, t)\right), \psi\left(\rho_{+}(x, t)\right)\right) .
\end{gathered}
$$


Filippov [3] shows that for every $x_{0}$, there exists a Lipschitz function $x(\cdot)$ such that $x\left(t_{0}\right)=x_{0}$, and

$$
\frac{d x}{d t} \in[L(x(t), t), U(x(t), t)]
$$

for almost all $t$.

For our purposes we need a solution such that for almost all $t$,

$$
\frac{d x}{d t} \in[l(x(t), t), u(x(t), t)] .
$$

Note that since $l$ and $u$ are well defined on the complement of a set of zero 1-dimensional Hausdorff measure, $l(x(t), t)$ and $u(x(t), t)$ are well defined for almost all $t$.

The following lemma implies that (5.8) always implies (5.9).

Lemma 5.1. Suppose $x:[0, T] \rightarrow \mathbb{R}$ is any Lipschitz function. Then for almost all $t$,

$$
\begin{aligned}
& U(x(t), t)=u(x(t), t), \\
& L(x(t), t)=l(x(t), t) .
\end{aligned}
$$

Proof. Step1: Since $b$ is in BV, we have

$$
\lim _{\delta \rightarrow 0} \frac{1}{\pi \delta^{2}} \int_{B_{\delta}(x, t)} b(y, s) d y d s=\frac{1}{2}(u(x, t)+l(x, t))
$$

for every $(x, t)$ that is either a point of approximate continuity or approximate jump discontinuity (see [18]). In particular the set of points at which (5.11) does not hold is of zero 1-dimensional Hausdorff measure. Therefore

$$
\lim _{\delta \rightarrow 0} \frac{1}{\pi \delta^{2}} \int_{B_{\delta}(x(t), t)} b(y, s) d y d s=\frac{1}{2}(u(x(t), t)+l(x(t), t)) .
$$

for almost all $t$.

By boundedness of $b$ we have

$$
\lim _{\delta \rightarrow 0} \int_{t_{1}}^{t_{2}} \frac{1}{\pi \delta^{2}} \int_{B_{\delta}(x(t), t)} b(y, s) d y d s d t=\frac{1}{2} \int_{t_{1}}^{t_{2}}(u(x(t), t)+l(x(t), t)) d t .
$$

Step 2: Let $A$ denote the left-hand side of (5.12). By Fubini's theorem

where

$$
A=\lim _{\delta \rightarrow 0} \int_{\mathbb{R}^{2}} b(z) H_{\delta}(z) d z,
$$

$$
H_{\delta}(z)=\frac{1}{\pi \delta^{2}}\left|\left\{t \in\left(t_{1}, t_{2}\right): z \in B_{\delta}(x(t), t)\right\}\right|,
$$

where $|\cdot|$ denotes the Lebesgue measure.

Let $\Gamma_{0}=\left\{(x(t), t): t_{1} \leqq t \leqq t_{2}\right\}$, and

$$
\Gamma_{\delta}=\left\{z: H_{\delta}(z) \neq 0\right\} .
$$

$\Gamma_{\delta}$ is simply the set of points in the $\delta$-vicinity of the set $\Gamma_{0}$. We can write $\Gamma_{\delta}$ as the union of $x$-intervals $I_{\delta}(t)$ for each $t \in\left[t_{1}(\delta), t_{2}(\delta)\right]$, where $\left[t_{1}(\delta), t_{2}(\delta)\right]$ is the projection of the set $\Gamma_{\delta}$ on the $t$-axis. Since $t_{1}(\delta), t_{2}(\delta)$ are close to $t_{1}$ and $t_{2}$ respectively, 
we have

$$
A=\lim _{\delta \rightarrow 0} \int_{t_{1}}^{t_{2}} \int_{I_{\delta}(t)} b(x, t) H_{\delta}(x, t) d x d t
$$

Step 3: The midpoint of each interval $I_{\delta}(t)$ is the point $x(t)$. For almost all $t, b(\cdot, t)$ is in $\mathrm{BV}$, and the left-hand and right-hand limits exists at every point $x$. One of the limit is $L(x, t)$, and the other is $U(x, t)$. We can write $I_{\delta}(t)=I_{\delta}^{+}(t) \cup I_{\delta}^{-}(t)$, where

$$
I_{\delta}^{-}(t)=\left\{x \in I_{\delta}(t): x \leqq x(t)\right\}, I_{\delta}^{+}(t)=I_{\delta}(t)-I_{\delta}^{-}(t) .
$$

Therefore

$$
\int_{I_{\delta}(t)} H_{\delta}(x, t) b(x, t) d x=U(x(t), t) H_{\delta}^{+}(x(t), t)+L(x(t), t) H_{\delta}^{-}(x(t), t)+o(1),
$$

where

$$
H_{\delta}^{ \pm}(x(t), t)=\int_{I_{\delta}^{ \pm}(t)} H_{\delta}(x, t) d x .
$$

Step 4: The value of $H_{\delta}^{ \pm}$depend on $x$ only. They are independent of the function $b$. In particular if $b$ is a piecewise smooth function, we have (5.10) and if $\lim _{\delta \rightarrow 0}$ $H_{\delta}^{ \pm}(x(t), t)=\alpha^{ \pm}(t)$, we have

$$
\alpha^{+}(t) U(x(t), t)+\alpha^{-}(t) L(x(t), t)=\frac{1}{2}(U(x(t), t)+L(x(t), t)) .
$$

Since $b$ can be any piecewise smooth function in this equality, we conclude $\alpha^{ \pm}(t)=\frac{1}{2}$.

Final Step: From (5.12), (5.13) and the previous step, we learn that

$$
U(x(t), t)+L(x(t), t)=l(x(t), t)+u(x(t), t)
$$

for almost all $t$. But we can repeat the above argument for the function $\hat{b}(x, t)=(b(x, t))^{2}$ and obtain

$$
(U(x(t), t))^{2}+(L(x(t), t))^{2}=(l(x(t), t))^{2}+(u(x(t), t))^{2}
$$

for almost all $t$. This and (5.14) imply (5.10).

The overall picture of the geometric structure of a BV function is the following. Most points in the set $\mathbb{R}_{+}^{2}$ are either points of approximate continuity or first order jump discontinuity. The set of points of approximate jump discontinuity can be embedded in a countable union of the rectifiable curves.

When $\rho$ is the entropy solution of (5.4), the set of points of jump discontinuity $\Gamma$ is called the shock set. At almost all points of $\Gamma$, (unit) normal vectors $v$ can be defined and if $v=\left(v^{1}, v^{2}\right)$, we have

$$
v^{1}\left(\rho_{+}-\rho_{-}\right)+v^{2}\left(h\left(\rho_{+}\right)-h\left(\rho_{-}\right)\right)=0 .
$$

In particular if $x(t)$ in any Lipschitz function, then for almost all $t$ in the set

we have

$$
\{t:(x(t), t) \in \Gamma\}
$$

$$
\frac{d x}{d t}=\frac{h\left(\rho_{+}(x(t), t)\right)-h\left(\rho_{-}(x(t), t)\right)}{\rho_{+}(x(t), t)-\rho_{-}(x(t), t)} .
$$


Our first theorem of this section asserts that a solution of (5.1) would avoid the shock sets if the density $\rho$ is nonzero. Recall that, by Lemma 5.1, $\rho^{ \pm}$and $\rho_{ \pm}$can be used interchangeably.

Theorem 5.2. Suppose $x$ is a solution to (5.1). Then for almost all $t$, either $\rho^{+}(x(t), t)=$ $\rho^{-}(x(t), t)$ or $\rho^{+}(x(t), t) \neq \rho^{-}(x(t), t)$ and $\rho^{+}(x(t), t) \rho^{-}(x(t), t)=0$.

Proof. Since $\rho$ is in BV, for almost all $t$, either $\rho^{+}(x(t), t)=\rho^{-}(x(t), t)$, or $(x(t), t) \in \Gamma$. On the other hand if $\rho^{+}, \rho^{-}$are two distinct positive constants, then

where

$$
\left(\psi\left(\rho^{+}\right)-c\right)\left(\psi\left(\rho^{-}\right)-c\right)<0,
$$

$$
c=\frac{h\left(\rho^{+}\right)-h\left(\rho^{-}\right)}{\rho^{+}-\rho^{-}} .
$$

Therefore if $\rho^{+}(x(t), t) \rho^{-}(x(t), t) \neq 0$, then

$$
\frac{h\left(\rho^{+}(x(t), t)\right)-h\left(\rho^{-}(x(t), t)\right)}{\rho^{+}(x(t), t)-\rho^{-}(x(t), t)} \notin[l(x(t), t), u(x(t), t)] .
$$

Thus if $\rho^{-}(x(t), t) \rho^{+}(x(t), t) \neq 0$, we have $\rho^{+}(x(t), t)=\rho^{-}(x(t), t)$ and this completes the proof.

An easy consequence of the above theorem is the following. If $\rho^{+}(x(t), t) \neq \rho^{-}(x(t), t)$, then either $\rho^{+}(x(t), t)=0$ or $\rho^{-}(x(t) t, t)=0$. If, for example, we assume $\rho^{-}(x(t), t)=0$, then for almost all such $t$ we have

$$
\frac{d x}{d t}=\frac{h\left(\rho^{+}(x(t), t)\right)-h\left(\rho^{-}(x(t), t)\right)}{\rho^{+}(x(t), t)-\rho^{-}(x(t), t)}=\psi\left(\rho^{+}(x(t), t)\right) .
$$

The following theorem plays a central role in the proof of our uniqueness results. Note by Lemma 5.9 below, the left-hand side of (5.5) is continuous in $t$.

Theorem 5.3. Let $m$ be a weak solution of (5.2) such that $0 \leqq m(x, t) \leqq \rho(x, t)$ for almost all $(x, t)$. Suppose $x(t)$ is a Filippov solution of (5.1). Then

for all $t$.

$$
\int_{-\infty}^{x(t)} m(y, t) d y=\int_{-\infty}^{x\left(t_{0}\right)} m\left(y, t_{0}\right) d y
$$

Proof. Step 1: For every test function $J$ that vanishes at $t=0$, we have

$$
\int_{0}^{\infty} \int\left\{J_{t}(x, t) m(x, t)+J_{x}(x, t) b(x, t) m(x, t)\right\} d x d t=0 .
$$

By approximation (5.15) holds for every Lipschitz function $J$.

Let $\xi$ be a smooth nonnegative function of compact support with $\int \xi(\rho) d \rho=1$. Choose $k$ such that $k^{\prime}=\xi$. Set $J(x, t)=\varphi(t) k\left(\frac{x-x(t)}{\varepsilon}\right)$, where $\varphi$ is a smooth function of compact support in $(0, \infty)$. We then have

$$
\begin{aligned}
J_{t}+b J_{x}= & \varphi^{\prime}(t) k\left(\frac{x-x(t)}{\varepsilon}\right)-\frac{1}{\varepsilon} \varphi(t) \xi\left(\frac{x-x(t)}{\varepsilon}\right) \frac{d x}{d t} \\
& +\frac{1}{\varepsilon} \varphi(t) \xi\left(\frac{x-x(t)}{\varepsilon}\right) b(x, t)
\end{aligned}
$$


for every point $t$ at which $\frac{d x}{d t}$ exists. Therefore (5.15) and Theorem (5.2) imply

$$
\begin{aligned}
\iint & \varphi^{\prime}(t) k\left(\frac{x-x(t)}{\varepsilon}\right) m(x, t) d x d t \\
= & \int_{A} \varphi(t) \int \frac{1}{\varepsilon} \xi\left(\frac{x-x(t)}{\varepsilon}\right)(b(x(t), t)-b(x, t)) m(x, t) d x d t \\
& \quad+\int_{B} \varphi(t) \int \frac{1}{\varepsilon} \xi\left(\frac{x-x(t)}{\varepsilon}\right)\left(\psi\left(\rho^{ \pm}(x(t), t)\right)-b(x, t)\right) m(x, t) d x d t \\
= & I_{\varepsilon}+I I_{\varepsilon},
\end{aligned}
$$

where $A=\left\{t: \rho^{+}(x(t), t)=\rho^{-}(x(t), t)\right\}$ and $B=(0, \infty)-A$.

Step 2: First we show $\lim _{\varepsilon \rightarrow 0} I_{\varepsilon}=0$. For this we estimate

$$
\begin{aligned}
I_{\varepsilon} & \leqq \int_{A} \varphi(t) \int \frac{1}{\varepsilon} \xi\left(\frac{x-x(t)}{\varepsilon}\right)|b(x(t), t)-b(x, t)| m(x, t) d x d t \\
& =\int_{A} \varphi(t) r_{\varepsilon}(t) d t
\end{aligned}
$$

and by the Bounded Convergence Theorem, it suffices to show

$$
\lim _{\varepsilon \rightarrow 0} r_{\varepsilon}(t)=0
$$

for almost every $t \in A$.

If $t \in A$, the function $b(x, t)$ is continuous as $x=x(t)$, which implies (5.17).

Step 3: If $t \notin A$, we have either $\rho^{-}(x(t), t)=0$ or $\rho^{+}(x(t), t)=0$. Let us assume $\rho^{-}(x(t), t)=0$, and $\rho^{+}(x(t), t)>0$. Since $m(x, t) \leqq \rho(x, t)$, we also have $m^{-}(x(t), t)=0$, in the sense that the $\lim _{y \uparrow x(t)} m(y, t)$ exists and is zero. Here some care is necessary because $m$ is merely a bounded measurable function. For almost all $t, m(x, t)$ is well defined as a function of $x$ and we can choose a version of $m(x, t)$ that is pointwise less than $\rho(x, t)$ for almost all $t$.

Now for each $t \in B$, we write

$$
\int \frac{1}{\varepsilon} \xi\left(\frac{x-x(t)}{\varepsilon}\right)\left(\psi\left(\rho^{+}(x(t), t)\right)-b(x, t)\right) m(x, t) d x=\int_{-\infty}^{x(t)}+\int_{x(t)}^{+\infty} .
$$

The limit of the first term is zero because $\lim _{y \uparrow x(t)} m(y, t)=0$. The limit of the second term is also zero because $\lim _{y \downarrow x(t)} b(x, t)=\psi\left(\rho^{+}(x(t), t)\right)$.

Final Step: From(5.16) and the previous step, we conclude that for every smooth $\varphi$ with compact support in $(0, \infty)$, we have

$$
\lim _{\varepsilon \rightarrow 0} \iint \varphi^{\prime}(t) k\left(\frac{x-x(t)}{\varepsilon}\right) m(x, t) d x d t=0 .
$$

On the other hand

$$
\lim _{\varepsilon \rightarrow 0} \int k\left(\frac{x-x(t)}{\varepsilon}\right) m(x, t) d x=\int_{-\infty}^{x(t)} m(x, t) d x
$$


because $m$ is bounded. Thus

is independent of $t$.

$$
\int_{-\infty}^{x(t)} m(x, t) d x
$$

Remark 5.4. If the initial density $\rho_{0}$ is positive, then the density stays positive for all time. For example suppose there exists a continuous function $f$ such that

$$
\rho_{0}(x) \geqq f(x)
$$

and $f(x)>0$ for all $x$. Then by finite speed of propagation, it is not hard to show that $\rho(x, t)>0$ for almost all $(x, t)$. In this case if $x(t)$ is a solution to $(5.1)$, we have $\rho^{+}(x(t), t)=\rho^{-}(x(t), t)$ for almost all $t$. (5.1).

The next theorem settles the uniqueness question for the initial value problem

If the density $\rho$ is positive, then we can apply (5.5) with $m=\rho$, and deduce that $x(t)$ can be determined uniquely in terms of $x(0)$.

For general $\rho$, it is necessary to use Oleinik's criterion on the nature of the discontinuity of $\rho$.

So for we know that the speed of the shock curve is given by $\left(h\left(\rho_{+}\right)-h\left(\rho_{-}\right)\right)$/ $\left(\rho_{+}-\rho_{-}\right)$.

Since $\rho$ is an entropy solution, Oleinik's criterion holds: the graph of $h$ lies below the chord joining $\left(\rho_{+}, h\left(\rho_{+}\right)\right)$to $\left(\rho_{-}, h\left(\rho_{-}\right)\right)$whenever $\rho_{-}>\rho_{+}$, and above the chord whenever $\rho_{-}<\rho_{+}$.

In particular we have

$$
\begin{aligned}
& \text { if } \rho_{+}=0<\rho_{-} \text {then } \psi(0)=\lim _{\rho \rightarrow 0} \frac{h(\rho)}{\rho} \leqq \frac{h\left(\rho_{-}\right)}{\rho_{-}}, \text {and } \\
& \text { if } \rho_{-}=0<\rho_{+} \text {then } \psi(0) \geqq \frac{h\left(\rho_{+}\right)}{\rho_{+}} .
\end{aligned}
$$

This property can be utilized to prove uniqueness even if the density $\rho$ vanishes on some set of positive Lebesgue measure.

Theorem 5.5. The initial value problem

$$
\left\{\begin{array}{l}
\frac{d x}{d t}=b(x, t) \quad t>t_{0} \\
x\left(t_{0}\right)=x_{0}
\end{array}\right.
$$

has at most one solution in Filippov sense.

Proof. Suppose $x, y$ are two solutions to (5.19). Let

$$
I=\left\{t>t_{0}: x(t) \neq y(t)\right\} .
$$

We want to show $I$ is empty. If $I$ is not empty, there exists an open interval $I_{0}=\left(t_{1}, t_{2}\right)$ such that $x\left(t_{1}\right)=y\left(t_{1}\right)$, and $x(t) \neq y(t)$ for all $t \in I_{0}$. Without loss of generality, we assume $x(t)<y(t)$ for every $t \in I_{0}$.

On the other hand, (5.5) implies

$$
\int_{x(t)}^{y(t)} \rho(x, t) d x=\int_{x\left(t_{1}\right)}^{y\left(t_{1}\right)} \rho\left(x, t_{1}\right) d x=0
$$


for every $t \in I_{0}$. Here we use the fact that $\rho$ solves (5.2) with the initial condition $m_{0}=\rho_{0}$.

Equation (5.20) implies $\rho(x, t)=0$ whenever $(x, t) \in D$, where

$$
D=\left\{(x, t): t_{1}<t<t_{2}, x(t)<x<y(t)\right\} .
$$

Pick a point $(z, s)$ in $D$ and let $\tau$ denote the line of slope $\psi(0)$ emanating from the point $(z, s)$. More precisely,

$$
\tau=\{(\gamma(t), t): t<s\}
$$

for some $\gamma$ such that $\gamma(s)=z$, and $\frac{d \gamma}{d t}=\psi(0)$. Therefore $\gamma$ is a solution to (5.1) as long as $(\gamma(t), t)$ stays in the set $D$.

The line $\tau$ intersects the boundary curve of $D$ at some point. In other words, there exists $t^{*} \in\left[t_{1}, s\right)$ such that either $\gamma\left(t^{*}\right)=x\left(t^{*}\right)$ or $\gamma\left(t^{*}\right)=y\left(t^{*}\right)$. We may assume $x$ is differentiable at $t^{*}$, if not we choose another point $\left(z^{\prime}, s^{\prime}\right) \in D$ that is close to $(z, s)$, and the corresponding line $\tau$ would intersect the boundary of $D$ at a differentiable point. We also assume $\gamma\left(t^{*}\right)=x\left(t^{*}\right)$ because the other case can be treated in the same way.

Since the line $\tau$ lies in the region $D$, and $x(t) \leqq y(t)$ for every $t \in\left[t_{1}, t_{2}\right]$, we have

$$
\frac{d x}{d t}\left(t^{*}\right)<\frac{d \gamma}{d t}\left(t^{*}\right)=\psi(0)
$$

But

$$
\frac{d x}{d t}\left(t^{*}\right)=\frac{h\left(\rho_{-}\left(x\left(t^{*}\right), t^{*}\right)\right)}{\rho_{-}\left(x\left(t^{*}\right), t^{*}\right)}
$$

and $\rho_{+}\left(x\left(t^{*}\right), t^{*}\right)=0$ which is in contradiction with (5.18). Thus $I=\emptyset$ and $x(t) \equiv y(t)$.

Remark 5.6. If the density $\rho$ is positive almost everywhere, we also have a unique solution to the terminal value problem

$$
\left\{\begin{array}{l}
\frac{d x}{d t}=b(x(t), t) \quad 0<t<t_{0} \\
x\left(t_{0}\right)=y_{0} .
\end{array}\right.
$$

Indeed the positivity of $\rho$ and

$$
\int_{-\infty}^{x(t)} \rho(x, t) d x=\int_{-\infty}^{x\left(t_{0}\right)} \rho\left(x, t_{0}\right)
$$

allow us to uniquely determine $x(t)$ in terms of $x\left(t_{0}\right)$.

The following example (part a) implies that (5.20) may have more than one solution if $\rho$ vanishes on a set of positive Lebesgue measure.

Example 5.7. Let $h(\rho)=\frac{1}{2} \rho^{2}$.

(a) Suppose

$$
\rho(x, t)= \begin{cases}1 & \text { if } x<t / 2, \text { and } \\ 0 & \text { otherwise }\end{cases}
$$


Then if $x(0)=x_{0} \leqq 0$, the unique solution of $(5.1)$ is $x(t)=x_{0}+\frac{1}{2} t$. If however $x_{0}>0$, then

$$
x(t)= \begin{cases}x_{0} & t<2 x_{0}, \text { and } \\ \frac{1}{2} t & t \geqq 2 x_{0} .\end{cases}
$$

Therefore, solutions starting from positive $x_{0}$ coincide on the line $x=\frac{1}{2} t$ for $t \geqq 2 x_{0}$.

(b) Suppose

$$
\rho(x, t)= \begin{cases}0 & \text { if } x<0, \\ \frac{x}{t} & \text { if } 0<x<t, \text { and } \\ 1 & \text { if } x>t .\end{cases}
$$

Then for every $x_{0}>0$, the solution of $(5.1)$ is

$$
x(t)= \begin{cases}x_{0}+\frac{1}{2} t & \text { if } t<2 x_{0}, \text { and } \\ \sqrt{2 x_{0} t} & \text { if } t \geqq 2 x_{0} .\end{cases}
$$

We now address the uniqueness question for (5.2).

Theorem 5.8. There exists at most one distributional solution $m$ to (5.2) such that

$$
0 \leqq m(x, t) \leqq \rho(x, t) .
$$

Proof. Let $x(t)$ be a solution to the terminal value problem (5.20). It follows from Theorem 4 of [3] that such solution exists. By Theorem 5.3, the expression $\int_{-\infty}^{x(t)} m(y, t) d y$ is independent of $t$. On the other hand we show in Lemma 5.9 below that the function $G$

$$
G(x, t):=\int_{-\infty}^{x} m(y, t) d y
$$

is jointly continuous. Therefore for every $t<t_{0}$,

$$
\int_{-\infty}^{y_{0}} m\left(y, t_{0}\right) d y=\int_{-\infty}^{x(t)} m(y, t) d y
$$

Since $x(\cdot)$ is Lipschitz, we can pass to the limit $t \rightarrow 0$,

$$
\int_{-\infty}^{y_{0}} m\left(y, t_{0}\right) d y=\int_{-\infty}^{x(0)} m_{0}(y) d y .
$$

Here we used the fact that $G(x, 0)=\int_{-\infty}^{x} m_{0}(y) d y$.

Now if $m_{0} \equiv 0$ then

$$
\int_{-\infty}^{y_{0}} m\left(y, t_{0}\right) d y=0
$$

for every $t_{0}$ and $y_{0}$, which in turn implies $m \equiv 0$.

Lemma 5.9. The function $G$ defined by (5.22) is Lipschitz in $x$ and $t$. 
Proof. Since $m$ is bounded, $G$ is Lipschitz in $x$. Formally we have

$$
\begin{aligned}
\frac{\partial G}{\partial t}(x, t) & =\int_{-\infty}^{x} m_{t}(y, t) d y \\
& =-\int_{-\infty}^{x}(b m)_{x} \\
& =-b(x, t) m(x, t) .
\end{aligned}
$$

It is not hard to show that in fact $G$ is weakly differentiable in $t$ with a derivative equal to $-b m$. Since both $b$ and $m$ are bounded, $G$ is also Lipschitz in $t$.

We end this section by giving a recipe for constructing the solutions of the PDE (5.2) in terms of the solution of the ODE (5.1).

Let $x\left(t, x_{0}\right)=x\left(t, x_{0} ; t_{0}\right)$ denote the unique solution of $(5.1)$.

Theorem 5.10. Let $m(x, t)$ be a weak solution of (5.2) satisfying

$$
0 \leqq m(x, t) \leqq \rho(x, t)
$$

for almost all $t \geqq t_{0}$. Then for every measurable $f$

$$
\int f\left(x\left(t, a ; t_{0}\right)\right) m_{0}(a) d a=\int f(x) m(x, t) d x
$$

holds for almost all $t$.

Proof. It is shown in Fillipov [3] that for each point $\left(x_{0}, t_{0}\right)$ there exist two solutions

$$
\begin{aligned}
& y^{-}(t)=y^{-}\left(t, x_{0} ; t_{0}\right), \\
& y^{+}(t)=y^{+}\left(t, x_{0} ; t_{0}\right),
\end{aligned}
$$

such that $y^{ \pm}\left(t_{0}\right)=x_{0}$, and every other solution passing through $\left(x_{0}, t_{0}\right)$ is between $y^{-}$and $y^{+}$. In other words if $y$ is any solution of (5.1), then

$$
y^{-}\left(t, x_{0} ; t_{0}\right) \leqq y(t) \leqq y^{+}\left(t, x_{0} ; t_{0}\right) .
$$

Now if $x_{1}$ is greater than $x_{0}$, we have

$$
x_{0} \leqq x(t, a) \leqq x_{1} \quad \text { if and only if } y^{-}\left(t_{0}, x_{1} ; t\right) \leqq a \leqq y^{+}\left(t_{0}, x_{2} ; t\right)
$$

and

$$
x\left(t, y^{-}\left(t_{0}, x_{1} ; t\right)\right)=x_{0}, x\left(t, y^{+}\left(t_{0}, x_{1} ; t\right)\right)=x_{1} .
$$

Thus

$$
\begin{aligned}
\int_{x_{0}}^{x_{1}} m(y, t) d y & =\int_{x\left(t, y^{-}\left(t_{0}, x_{1} ; t\right)\right)}^{x\left(t, y^{+}\left(t_{0}, x_{1} ; t\right)\right)} m(y, t) d y \\
& =\int_{y^{-}\left(t_{0}, x_{1} ; t\right)}^{y^{+}\left(t_{0}, x_{1} ; t\right)} m_{0}(a) d a \quad \text { by }(5.5)
\end{aligned}
$$

which implies (5.23) for $f=\mathbb{1}_{\left[x_{0}, x_{1}\right]}$. 


\section{Proof of Main Theorems}

Proof of Theorem 2.7. Step 1: In this step we established the tightness of the sequence $Q^{L}$.

Let $c$ be a fixed vector in $\mathbb{R}^{d}$. Let $f(x)=\frac{1}{L} x_{1} \cdot c$ where $\mathbf{x}=\left(x_{1} \ldots x_{N}\right)$. Then

$$
\frac{1}{L} x_{1}(t L) \cdot c=\frac{1}{L} x_{1}(0) \cdot c+\int_{0}^{t L} \hat{\mathscr{L}} f(\mathbf{x}(s)) d s+M_{t L},
$$

where $M$ is a martingale with quadratic variation

$$
E^{L} M_{t L}^{2}=\int_{0}^{t L}\left(\hat{\mathscr{L}} f^{2}-2 f \hat{\mathscr{L}} f\right)(\mathbf{x}(s)) d s .
$$

Here $E^{L}$ denotes the expectation with respect to $P^{L}$.

A straightforward computation reveals

$$
\begin{aligned}
\hat{\mathscr{L}} f(\mathbf{x}) & =\sum_{z}(z \cdot c) p(z) \frac{b\left(\eta\left(\mathbf{x} ; x_{1}\right), \eta\left(\mathbf{x} ; x_{1}+z\right)\right)}{\eta\left(\mathbf{x} ; x_{1}\right)} \\
\left(\hat{\mathscr{L}} f^{2}-2 f \hat{\mathscr{L}} f\right)(\mathbf{x}) & =\frac{1}{L} \sum_{z}(z \cdot c)^{2} p(z) \frac{b\left(\eta\left(\mathbf{x} ; x_{1}\right), \eta\left(\mathbf{x} ; x_{1}+z\right)\right)}{\eta\left(\mathbf{x} ; x_{1}\right)} .
\end{aligned}
$$

Therefore

$$
E^{L} M_{t L}^{2} \leqq \frac{\text { const. }}{L}
$$

and by Doob's inequality we even have

$$
E^{L} \sup _{0 \leqq t \leqq t_{0}} M_{t L}^{2} \leqq \frac{\text { const. }}{L}
$$

for every $t_{0}$. Now this and (6.1) imply

$$
\lim _{\delta \rightarrow 0} \limsup _{L \rightarrow \infty} Q^{L}\left(\sup _{|t-s|<\delta}\left|y_{1}(t)-y_{1}(s)\right|>\varepsilon\right)=0
$$

for every positive $\varepsilon$. Recall that $Q^{L}$ is a probability measure on the space of configurations $\mathbf{y}=\left(y_{1}, \ldots, y_{N}\right)$, where each $y_{i} \in \mathscr{D}\left([0, T] ; \mathbb{R}^{d}\right)$.

It is well known that the tightness of $y_{1, L}(t)=\frac{1}{L} x_{1}(t L)$ is equivalent to the tightness of

$$
\bar{X}_{L}=\frac{1}{N} \sum_{i=1}^{N} \delta_{y_{i, L}}
$$

as a sequence of $\mathscr{M}(\mathscr{D})$-valued random variables. See for example Sznitman [17].

Step 2. By tightness we only need to identify the finite-dimensional distribution of the limit points. This can be done by establishing (2.21) for functions $J$ of the form

$$
J(y)=J_{1}\left(y\left(t_{1}\right)\right) J_{2}\left(y\left(t_{2}\right)\right) \ldots J_{k}\left(y\left(t_{k}\right)\right),
$$

where $0 \leqq t_{1}<t_{2}<\cdots<t_{k}$, and $J_{1} \ldots J_{k}: \mathbb{R}^{d} \rightarrow \mathbb{R}$. Let

$$
\left\langle\bar{X}_{L}, J\right\rangle:=\frac{1}{N} \sum_{i=1}^{N} J\left(y_{i, L}\right)
$$


for a $J$ of the form (6.3). If $k=1,\left\langle\bar{X}_{L}, J\right\rangle$ converges in measure to

$$
\int J_{1}(x) \rho\left(x, t_{1}\right) d x \text {. }
$$

This follows from Theorem 2.2. Suppose $k=2, J_{2}$ is any continuous and $J_{1}(x)=$ $\mathbb{1}_{A_{1}}(y)$ for some measurable set $A_{1}$. Then $\left\langle\bar{X}_{L}, J\right\rangle$ converges to

$$
\int J_{2}(x) m_{2}\left(x, t_{2}\right) d x
$$

where $m_{2}(x, \cdot)$ is the unique weak solution of

$$
\begin{cases}\partial_{t} m_{2}+\left(b m_{2}\right)_{x}=0 & \text { for } t>t_{1} \\ m_{2}\left(x, t_{1}\right)=\rho\left(x, t_{1}\right) \mathbb{1}_{A_{1}}(x) & \end{cases}
$$

This follows from Theorem 3.1, Theorem 5.8, and the first paragraph of Sect. 3 .

Step 3: General $k$ can be treated inductively. Take

$$
J_{i}=\mathbb{1}_{A_{i}} \quad i=1, \ldots, k-1,
$$

where $A_{1} \ldots A_{k-1}$ are measurable subsets of $\mathbb{R}^{d}$. Suppose $J_{k}$ is any measurable function.

Let $m_{j}(x, t)$, for $t>t_{j-1}$, denote the density of particles that are in the set $A_{i}$ at time $t_{i}$ for $i=1, \ldots, j-1$. Suppose that we have already determined $m_{k-1}(x, t)$ for $t>t_{k-1}$. Then Theorem 3.1 can be applied to show that $\left\langle\bar{X}_{L}, J\right\rangle$ converges to

$$
\int m_{k}(x, t) J_{k}(x) d x,
$$

where $m_{k}$ is the unique weak solution of

$$
\begin{cases}\partial_{t} m_{k}+\left(b m_{k}\right)_{x}=0 & \text { for } t>t_{k-1} . \\ m_{k}\left(x, t_{k-1}\right)=m_{k-1}\left(x, t_{k-1}\right) \mathbb{1}_{A_{k-1}}(x) . & \end{cases}
$$

Final Step: We need to identify the limit points as $R$. Recall that $R$ is the law of $x(t, a ; 0)$ where $a$ is distributed according to the probability measure $c \rho_{0}(a) d a$. Also by uniqueness, we have the semigroup property

$$
x\left(t_{3}, a ; t_{1}\right)=x\left(t_{3}, x\left(t_{2}, a ; t_{1}\right) ; t_{2}\right),
$$

for $t_{1} \leqq t_{2} \leqq t_{3}$.

We start with $k=1$. By Theorem 5.10 we have

$$
\int J_{1}\left(x\left(t_{1}, a ; 0\right)\right) \rho_{0}(a) d a=\int J_{1}(x) \rho\left(x, t_{1}\right) d x
$$

because $\rho$ solves (5.1) with $t_{0}=0$ and $m_{0}=\rho_{0}$. This implies that the one-dimensional distribution of $x(t)$ with respect to $R$ has density $c \rho(x, t)$.

Now we consider the case $k=2$. We apply Theorem 5.10 and 6.3 to write

$$
\begin{aligned}
\int J_{1}\left(x\left(t_{1}, a ; 0\right)\right) J_{2}\left(x\left(t_{2}, a ; 0\right)\right) \rho_{0}(a) d a \\
\quad=\int J_{1}\left(x\left(t_{1}, a ; 0\right)\right) J_{2}\left(x\left(t_{2}, x\left(t_{1}, a ; 0\right) ; t_{1}\right)\right) \rho_{0}(a) d a \\
\quad=\int J_{1}(y) J_{2}\left(x\left(t_{2}, y ; t_{1}\right)\right) \rho\left(y, t_{1}\right) d y .
\end{aligned}
$$

Now suppose $J_{1}(y)=\mathbb{1}_{A_{1}}(y)$ and apply Theorem 5.10 once more

$$
=\int J_{2}(z) m_{2}\left(z, t_{2}\right) d z \text {. }
$$

The general $k$ can be treated inductively. 
Proof of Theorem 2.9. Step 1: We paint all the particles in the interval $([a L]-$ $\varepsilon L,[a L]+\varepsilon L)$ blue, and the remaining particles white. If $m_{\varepsilon}(x, t)$ denotes the density of the blue particles, then by Theorem 3.1

Let

$$
\left\{\begin{array}{l}
\partial_{t} m_{\varepsilon}+\left(b m_{\varepsilon}\right)_{x}=0 \\
m_{\varepsilon}(x, 0)=\rho_{0}(x) \mathbb{1}_{[a-\varepsilon, a+\varepsilon]}(x) .
\end{array}\right.
$$

$$
\begin{aligned}
& A_{\varepsilon}=\left\{(x, t): m_{\varepsilon}(x, t) \neq 0\right\} \\
& B_{\varepsilon}=\{(x, t): x(t, a-\varepsilon ; 0) \leqq x \leqq x(t, a+\varepsilon ; 0)\} .
\end{aligned}
$$

First we claim $B_{\varepsilon}-A_{\varepsilon}$ has zero Lebesgue measure. Indeed, by Theorem 5.10, $m_{\varepsilon}(x, t)$ satisfies

$$
\int_{0}^{\infty} \int_{a-\varepsilon}^{a+\varepsilon} f(x(t, z ; 0), t) \rho_{0}(z) d z d t=\int_{0}^{\infty} \int f(x, t) m_{\varepsilon}(x, t) d x d t
$$

for every measurable function $f$. On the other hand if $z \in[a-\varepsilon, a+\varepsilon]$ then

$$
x(t, a-\varepsilon ; 0) \leqq x(t, z ; 0) \leqq x(t, a+\varepsilon ; 0) .
$$

This implies that the left-hand side of (6.4) is zero is $f=\mathbb{1}_{B_{\varepsilon}^{c}}$. Therefore

$$
\iint_{B_{\varepsilon}^{c}} m_{\varepsilon}(x, t) d x d t=0 \text {. }
$$

This in turn implies $A_{\varepsilon} \subseteq B_{\varepsilon}$ (almost everywhere) because $m_{\varepsilon} \geqq 0$.

Step 2: Under Assumption 2.8 particles do not cross each other. On the other hand the particle densities on the left and right side of the tagged particle are positive. This implies that the macroscopic location of the tagged particle is always in the set $A_{\varepsilon}$. Thus it suffices to show

$$
\bigcap_{\varepsilon>0} B_{\varepsilon}=\{(x(t, a ; 0), t: t \geqq 0\} .
$$

Final Step: It suffices to show that for every $t_{0}$,

$$
\sup _{\varepsilon>0} x\left(t_{0}, a-\varepsilon\right)=\inf _{\varepsilon>0} x\left(t_{0}, a+\varepsilon\right)
$$

because $x\left(t_{0}, a-\varepsilon\right) \leqq x\left(t_{0}, a\right) \leqq x\left(t_{0}, a+\varepsilon\right)$. Suppose to the contrary there is some $z$ such that $z \neq x(t, \bar{a})$, and

$$
x\left(t_{0}, a-\varepsilon\right)<z<x\left(t_{0}, a+\varepsilon\right)
$$

for all $\varepsilon$. Let $y$ be a solution to the terminal value problem

$$
\left\{\begin{array}{l}
\frac{d y}{d t}=b(y, t) \quad 0 \leqq t<t_{0} \\
y\left(t_{0}\right)=z .
\end{array}\right.
$$

But $y(0) \neq a$ because $x \neq x(t, a)$. Therefore $y(0)<a-\varepsilon_{0}$ or $y(0)>a+\varepsilon_{0}$ for some $\varepsilon_{0}>0$. If, for example, $y(0)<a-\varepsilon_{0}$, then

$$
x(t, y(0))=z \leqq x\left(t, a-\varepsilon_{0}\right)
$$

contradiction. Thus (6.5) holds. 
We now discuss that how under Assumption 2.4, a variant of Theorem 2.7 holds. A review of the proof of Theorem 2.7 reveals that we can still repeat steps one to three. In order to complete the proof, what we really need is to uniquely identify the finite dimensional distribution of the process $\bar{X}_{L}$. For this it suffices to establish the uniqueness of the PDE (5.2) under the bound (2.15). In fact, this bound also holds for the function $b$.

Lemma 6.1. There exists a constant $C$ such that

$$
b(x+r \gamma, t)-b(x, t) \leqq C\left(1+\frac{1}{t}\right) r
$$

for almost all $t$ and $r$.

Proof. Since $\psi$ is smooth, it suffices to show $\psi$ is nondecreasing on the interval $[0, \infty)$. First we write

$$
0=h(0)=h(\rho)+(-\rho) h^{\prime}(\rho)+\frac{1}{2}(-\rho)^{2} h^{\prime \prime}(\theta)
$$

for some $\theta \in[0, \rho]$. Therefore

$$
h(\rho)-\rho h^{\prime}(\rho) \leqq 0
$$

On the other hand

$$
\psi^{\prime}(\rho)=\frac{\rho h^{\prime}(\rho)-h(\rho)}{\rho^{2}} \geqq 0
$$

for all $\rho>0$.

The following lemma will allow us to identify the limit points of the sequence $\bar{X}_{L}$ providing $h$ is uniformly convex.

Lemma 6.2. Suppose (6.6) holds. Then there is at most one solution to the PDE (3.2).

The proof of this lemma follows Theorem 16.11 of Smoller [15]. Note that Theorem 16.11 in [15] only asserts that there is at most one solution to the one conservation law (5.4) if $h$ is uniformly convex. However one can easily check that the proof of this theorem implies Lemma 6.2.

Acknowledgements. I wish to thank L.C. Evans for pointing out the relevance of Lemma 6.2 to this work. I am also grateful to S.R.S. Varadham from whom I learnt the proof of Lemma 4.1.

\section{References}

1. Cocozza, C.T.: Processus des misanthropes. Z. Wahrs. Verw. Gebiete 70, 509-523 (1985)

2. Dafermos, C.M.: Generalized characteristics and the structure of solutions of hyperbolic conservation laws. Indiana U. Math. J. 26, 1097-1119 (1977)

3. Filippov, A.F.: Differential equations with discontinuous right-hand side. Mat. Sbornik (N.S.) 51 (93), 99-128 (1960)

4. Guo, M.Z., Papanicolaou, G.C., Varadhan, S.R.S.: Nonlinear diffusion limit for a system with nearest neighbor interactions. Commun. Math. Phys. 118, 31-59 (1988)

5. Kipnis, C.: Central limit theorems for infinite series of queues and applications to simple exclusion. Ann. Probab., Vol 14, No. 2, 397-408, (1986)

6. Kipnis, C., Varadhan, S.R.S.: Central limit theorem for additive functionals for reversible Markov processes and applications to simple exclusions. Commun. Math. Phys. 104, 1-19 (1986) 
7. Kružkov, S.N.: First order quasilinear equations in several independent variables. Math. USSR-Sb 10, 217-243 (1970)

8. Liggett, T.M.: Interacting Particle Systems. Berlin, Heidelberg, New York: Springer, 1985

9. Quastel, J.: Diffusion of colour in the simple exclusion process. Comm. Pure Appl. Math.

10. Rezakhanlou, F.: Hydrodynamic limit for attractive particle systems on $\mathbb{Z}^{d}$. Commun. Math. Phys. 140, 417-448 (1991)

11. Rezakhanlou, F.: Propagation of chaos for symmetric simple exclusions. To appear in Comm. Pure Appl. Math.

12. Rezakhanlou, F.: Microscopic structure of shocks in one conservation laws, preprint

13. Saada, E.: A limit theorem for the position of a tagged particle in a simple exclusion process. Ann. Probab., Vol. 15, 375-381 (1987)

14. Saada, E.: Processus de zero-range avec particule marquée. Ann. Inst. Henri Poincaré, Vol 26, No. 1, 5-17 (1990)

15. Smoller, J.: Shock Waves and Reaction-Diffusion Equations. Berlin, Heidelberg, New York: Springer, 1980

16. Spohn, H.: Large Scale Dynamics for Interacting Particles. Berlin, Heidelberg, New York: Springer, 1991

17. Sznitman, A.: Topics in propagation of chaos. Lecture Notes in Mathematics 1464, Berlin, Heidelberg, NewYork: Springer, (1989)

18. Zeimer, W.: Weakly Differentiable Functions. Berlin, Heidelberg, NewYork: Springer, 1989

Communicated by J.L. Lebowitz 